Synthesis and characterization of a novel donor-acceptor-donor chiral inducer and application in electrochemical polymerization

Jiuchao Dong, Kohsuke Kawabata, and Hiromasa Goto*

J. Mater. Chem. C, 2015,3, 2024-2032.

\title{
Synthesis and characterization of a novel donor-acceptor-donor chiral inducer and application in electrochemical polymerization
}

Jiuchao Dong, Kohsuke Kawabata, and Hiromasa Goto*

Graduate School of Pure and Applied Sciences, Institute of Material Science, University of Tsukuba, Tsukuba, Ibaraki 305-8573, Japan

Correspondence to H. Goto, e-mail: gotoh@ims.tsukuba.ac.jp. FAX:+81-298-53-4490

\section{Highlight}

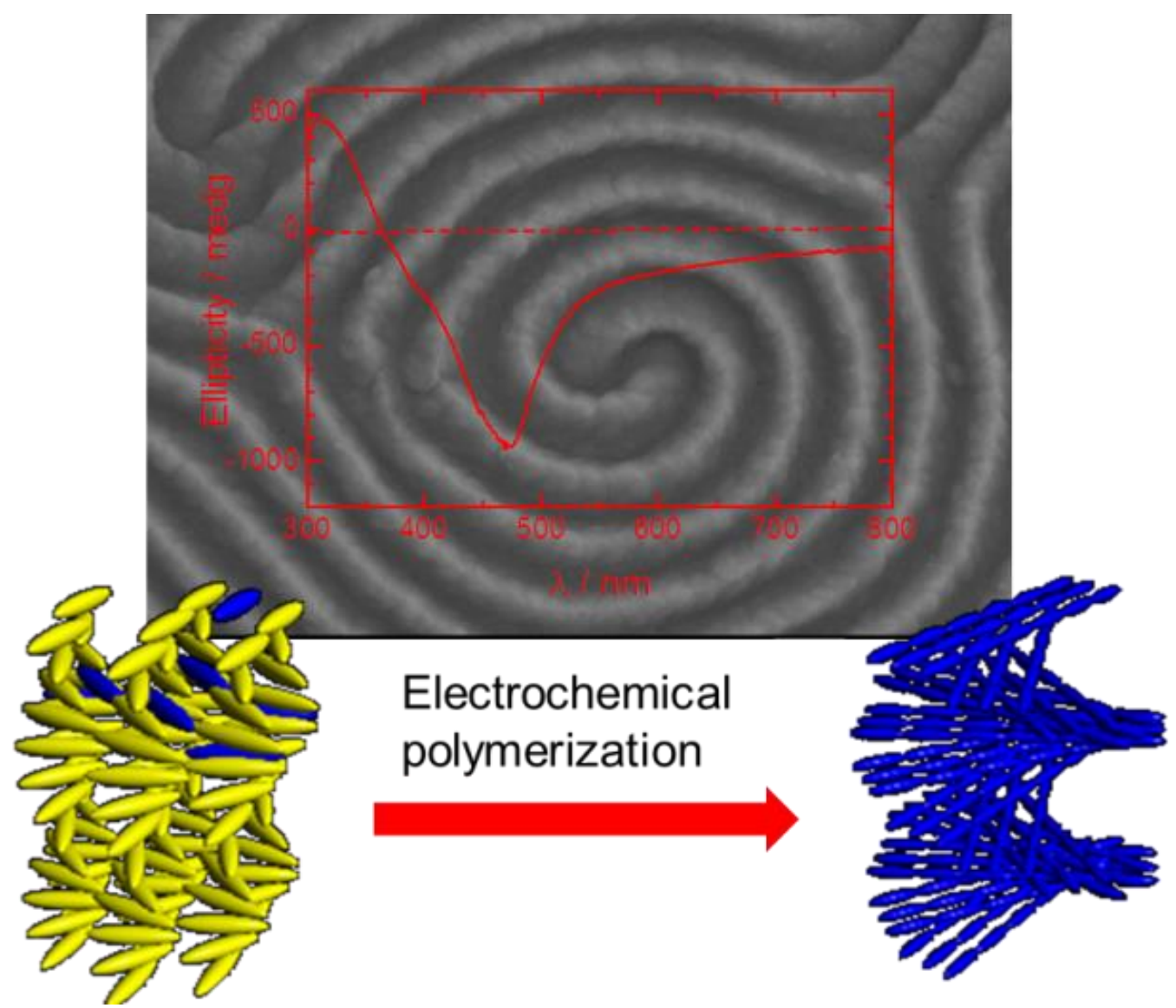

A novel chiral inducer was synthesized for obtaining a spirally arranged conjugated polymer without chiral monomers. 
Synthesis and characterization of a novel donor-acceptor-donor chiral inducer and application in electrochemical polymerization

Jiuchao Dong, Kohsuke Kawabata, and Hiromasa Goto*

J. Mater. Chem. C, 2015,3, 2024-2032.

\title{
Synthesis and characterization of a novel donor-acceptor-donor chiral inducer and application in electrochemical polymerization
}

Jiuchao Dong, Kohsuke Kawabata, and Hiromasa Goto*

Graduate School of Pure and Applied Sciences, Institute of Material Science, University

of Tsukuba, Tsukuba, Ibaraki 305-8573, Japan

Correspondence to H. Goto, e-mail: gotoh@ims.tsukuba.ac.jp. FAX:+81-298-53-4490

\section{Table of contents entry}

A novel chiral inducer was synthesized for obtaining a spirally arranged conjugated polymer without chiral monomers.

\begin{abstract}
A novel chiral inducer having donor-acceptor-donor structure was synthesized. Its rigid structure allows an increase of miscibility and helical twisting power for the host liquid crystal. The donor-acceptor-donor structure allows visible light absorption. Electrochemical polymerization of an achiral monomer was performed in a cholesteric liquid crystal induced by the inducer. The chiral inducer molecules alone showed right-handed helical aggregation in the solid state, while the inducer produces left-handed helical arrangement of cholesteric liquid crystal in a nematic liquid crystal. Resultant conjugated polymer films showed circular dichroism and left-handed helical aggregation. Film surfaces displayed periodic convex-concave structure that was transcribed from the fingerprint texture of the cholesteric liquid crystal electrolytes during the polymerization process.
\end{abstract}

\section{Introduction}

$\pi$-Conjugated polymers have been investigated for various applications: organic solar cells, ${ }^{1-2}$ organic field-effect transistors (OFETs) ${ }^{3-5}$ and organic light emitting diodes (OLEDs). ${ }^{6-7}$ Conjugated polymers can show anisotropic features such as electric conductivity along the main chain. Controlling the molecular arrangement is a significant issue in materials processing. Fortunately, electrochemical polymerization provides a simple and quick method for obtaining conjugated polymer films compared 
Synthesis and characterization of a novel donor-acceptor-donor chiral inducer and application in electrochemical polymerization

Jiuchao Dong, Kohsuke Kawabata, and Hiromasa Goto*

J. Mater. Chem. C, 2015,3, 2024-2032.

with chemical polymerization, both of which can be directly applied in OLED and OFET. $^{8-10}$ Electrochemical polymerization in liquid crystal (LC) electrolytes is a promising method for controlling the arrangement of conjugated polymers. Because of its fluidity, LC can be used as a reaction medium for electro-polymerization by mixing with a small amount of supporting salt and monomers. The chain propagation occurs along the director of the LCs. Therefore, various conjugated polymers with different structure arrangements can be obtained with electrochemical polymerization in a variety of LCs and monomers.

Our group has performed electrochemical polymerization in a smectic A LC state that was oriented by a magnetic field. ${ }^{11}$ The obtained polythiophene derivative films were uniaxially oriented at the macroscopic level. We also reported various conditions of electrochemical polymerization in cholesteric LC. ${ }^{12-16}$ In these cases, we obtained chiral conjugated polymer films by using an achiral monomer. The polymers transcribed the fingerprint texture of cholesteric LCs, and showed helical molecular arrangement and periodic concave-convex surface structure. Moreover, selective reflection of the films upon irradiation of light was observed. This results demonstrated that electrochemical polymerization in LC is an effective method for controlling molecular orientation and surface structure of resultant polymer films.

As for electrochemical polymerization in cholesteric LC, the molecular orientation and surface structure of polymer films are significantly affected by the helical architecture. Cholesteric LC can be obtained by adding chiral inducers to nematic LCs. The helical structure of cholesteric LC depends on the quantity and helical twisting power of chiral inducers. To obtain the expected helical structure, chiral inducers with high twisting helical power are required. In addition, the chiral inducer should have following features: (1) good inter-miscibility with the host nematic LC; (2) the blend LC (chiral inducer and nematic LC) shows stable cholesteric phase; (3) the LCs show wide temperature range of cholesteric phase. Furthermore, cholesteric LC phase temperature range is preferable at around room temperature for convenient operation of the electrochemical polymerization experiments.

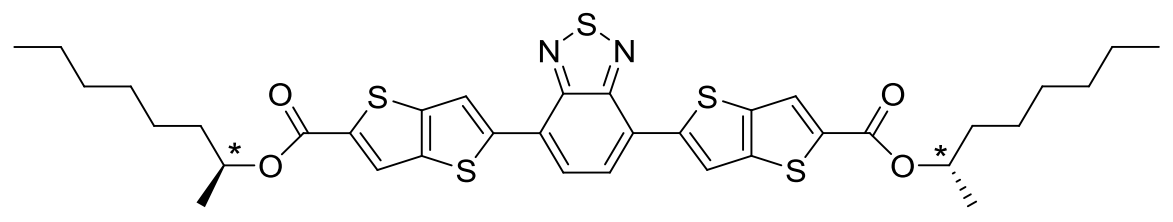

Fig. 1. Compound (S)-D8Tt-B*. 
Synthesis and characterization of a novel donor-acceptor-donor chiral inducer and application in electrochemical polymerization

Jiuchao Dong, Kohsuke Kawabata, and Hiromasa Goto*

J. Mater. Chem. C, 2015,3, 2024-2032.

In this work, we synthesized a novel chiral inducer for application in electrochemical polymerization (Fig. 1). The mesogenic core consists of thieno[3,2-b]thiophene and benzo[c][1,2,5]thiadiazole. This rod-like structure may increase the miscibility in the rod-shaped LC. Furthermore, this donor-acceptor-donor (D-A-D) structure shows optical absorption in the visible range. ${ }^{17-20}$ The characteristic optical absorption can be helpful for examining orientational properties of this chiral inducer with optical spectroscopy.

The chiral inducer abbreviated as $(S)$-D8Tt-B* was synthesized by the Mitsunobu reaction, and the Suzuki-Miyaura coupling reaction. Thermodynamic properties and optical properties of the resultant materials were investigated. Further, a mixture of $(S)-\mathrm{D} 8 \mathrm{Tt}-\mathrm{B}^{*}$ with 4-cyano-4'-hexylbiphenyl (6CB, nematic LC at room temperature) was prepared for investigating the chiral induction properties. Finally, electrochemical polymerization of monomers in a cholesteric LC electrolyte solution prepared by using the chiral inducer was carried out. Optical properties, surface structure and electrochemical properties of the resultant polymer films were examined.
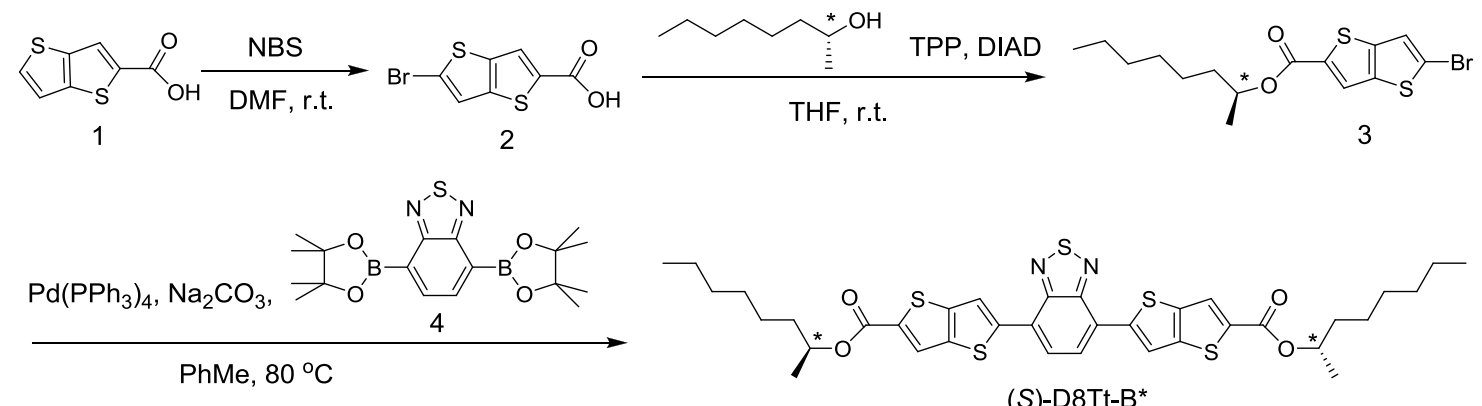

(S)-D8Tt-B*

Scheme 1. Synthetic route to compound (S)-D8Tt-B*.

\section{Experimental}

\section{Synthesis of chiral inducer $((S)$-D8Tt-B*)}

The synthetic route for the target compound is shown in Scheme 1. Thieno[3,2-b]thiophene-2-carboxylic acid $\mathbf{1}$ was brominated with $N$-bromosuccinimide (NBS) to yield compound 2 followed by the Mitsunobu reaction with (R)-(-)-2-octanol to afford the ester 3. Herein, the absolute configuration of the chiral center can be inverted during this $S_{N} 2$ reaction, according to the Walden inversion. Subsequent Suzuki-Miyaura coupling reaction between the esters $\mathbf{3}$ and compound $\mathbf{4}$ afforded target compound $(S)$-D8Tt-B*. Chemical structures of the compounds were confirmed with ${ }^{1} \mathrm{H}$ NMR. 
Synthesis and characterization of a novel donor-acceptor-donor chiral inducer and application in electrochemical polymerization

Jiuchao Dong, Kohsuke Kawabata, and Hiromasa Goto*

J. Mater. Chem. C, 2015,3, 2024-2032.

5-Bromo-thieno[3,2-b]thiophene-2-carboxylic acid(compound 2). To a solution of thieno[3,2-b]thiophene-2-carboxylic acid (3.68 g, $20 \quad \mathrm{mmol})$ in $N, N$-dimethylformamide (DMF, $66 \mathrm{~mL}$ ) was slowly added NBS (3.56 g, $20 \mathrm{mmol}$ ). The mixture was stirred at room temperature for $20 \mathrm{~h}$. After the reaction, $500 \mathrm{~mL}$ of water was poured into the mixture. The resultant white precipitate was filtered and washed with a large volume of water. A light-pink solid was obtained after drying in vacuum. Yield $=92 \%$ (4.812 g, $18.35 \mathrm{mmol}) .{ }^{1} \mathrm{H}$ NMR (400 MHz; DMSO-D6; TMS) $\delta 7.842$ (s, $1 \mathrm{H}), 8.464(\mathrm{~s}, 1 \mathrm{H}) .{ }^{13} \mathrm{C} \mathrm{NMR}\left(100 \mathrm{MHz} ; \mathrm{CDCl}_{3}\right.$; TMS ) $\delta 117.730,123.372$, 125.936, 138.927, 142.348, 163.297, 171.675.Elem. Anal.: Calcd for $\mathrm{C}_{7} \mathrm{H}_{3} \mathrm{BrO}_{2} \mathrm{~S}_{2}: \mathrm{C}$, 31.95; H, 1.15; N, 0.00. Found: C, 31.8; H, 0.88; N, 0.06.

(S)-Octan-2-yl 5-bromothieno[3,2-b]thiophene-2-carboxylate (compound 3). To a solution of 5-bromothieno[3,2-b]thiophene-2-carboxylic acid (0.412 g, $1.57 \mathrm{mmol})$, triphenylphosphine $(0.42 \mathrm{~g}, 1.6 \mathrm{mmol})$, and $(R)$-(-)-2-octanol $(0.208 \mathrm{~g}, 1.57 \mathrm{mmol})$ in tetrahydrofuran (THF) $(5 \mathrm{~mL})$ was added diisopropyl azodicarboxylate (40\% in toluene) $(0.84 \mathrm{~mL}, 1.6 \mathrm{mmol})$ dropwise at $0{ }^{\circ} \mathrm{C}$. After stirring for $22 \mathrm{~h}$, the solvent was evaporated. The crude products were extracted with diethyl ether, followed by drying in $\mathrm{MgSO}_{4}$, then purified by silica gel column chromatography (chloroform/hexane = $1 / 2)$ to afford a white solid $(0.515 \mathrm{~g}, 1.37 \mathrm{mmol}, 87 \%) .{ }^{1} \mathrm{H} \mathrm{NMR}\left(400 \mathrm{MHz} ; \mathrm{CDCl}_{3}\right.$; TMS) $\delta 0.859(\mathrm{t}, 3 \mathrm{H}, J=6.6 \mathrm{~Hz}), 1.281-1.405(\mathrm{~m}, 11 \mathrm{H}), 1.600-1.768(\mathrm{~m}, 2 \mathrm{H}), 5.081$ (sext, $1 \mathrm{H}, J=5.8 \mathrm{~Hz}), 7.292(\mathrm{~s}, 1 \mathrm{H}), 7.865(\mathrm{~s}, 1 \mathrm{H}) .{ }^{13} \mathrm{C} \mathrm{NMR}\left(100 \mathrm{MHz} ; \mathrm{CDCl}_{3} ; \mathrm{TMS}\right)$ $\delta$ 14.086, 20.091, 22.635, 25.371, 29.126, 31.766, 35.988, 72.721, 118.193, 122.415, 124.712, 135.139, 138.856, 142.659, 162.149.Elem. Anal.: Calcd for $\mathrm{C}_{15} \mathrm{H}_{19} \mathrm{BrO}_{2} \mathrm{~S}_{2}: \mathrm{C}$, 48.0; H, 5.10; N, 0.00. Found: C, 48.08; H, 4.89; N, 0.03.

\section{(S)-Dioctan-2-yl 5,5'-(benzo[c][1,2,5]thiadiazole-4,7-diyl)dithieno[3,2-b]thiophene}

-2-carboxylate $((S)$-D8Tt-B*). In an argon-flushed, two-neck, round-bottom flask, a mixture of $(S)$-octan-2-yl 5-bromothieno[3,2-b]thiophene-2-carboxylate $(0.257 \mathrm{~g}$, $0.685 \mathrm{mmol}), 4,7$-bis(4,4,5,5-tetramethyl-1,3,2-dioxaborolan-2-yl)benzo[c][1,2,5]thiadiazole (0.135 g, $0.35 \mathrm{mmol})$, tetrakis(triphenylphosphine)palladium(0) (0.032 g, 0.028 mmol), degassed $1 \mathrm{~mL}$ solution of $\mathrm{Na}_{2} \mathrm{CO}_{3}(0.217 \mathrm{~g}, 2.06 \mathrm{mmol})$ and toluene $(2 \mathrm{~mL})$ was heated at $80{ }^{\circ} \mathrm{C}$ and refluxed for $20 \mathrm{~h}$. After cooling, it was extracted with dichloromethane and dried over $\mathrm{MgSO}_{4}$. The solvent was removed with a rotary evaporator. The residue in the flask was purified by column chromatography (silica gel, hexane/chloroform=1:1), yielding a red solid $(0.186 \mathrm{~g}, 0.256 \mathrm{mmol}, 73 \%) .{ }^{1} \mathrm{H}$ $\operatorname{NMR}\left(400 \mathrm{MHz} ; \mathrm{CDCl}_{3}\right.$; TMS) $\delta 0.870(\mathrm{t}, 6 \mathrm{H}, J=6.6 \mathrm{~Hz}), 1.251-1.456(\mathrm{~m}, 22 \mathrm{H})$, 1.583-1.793 (m, 4H), $5.112(\mathrm{sext}, 2 \mathrm{H}, J=5.8 \mathrm{~Hz}), 7.924(\mathrm{~s}, 2 \mathrm{H}), 7.995$ (s, 2H), 8.503 (s, 
Synthesis and characterization of a novel donor-acceptor-donor chiral inducer and application in electrochemical polymerization

Jiuchao Dong, Kohsuke Kawabata, and Hiromasa Goto*

J. Mater. Chem. C, 2015,3, 2024-2032.

2H). ${ }^{13} \mathrm{C}$ NMR (100 MHz; $\mathrm{CDCl}_{3}$; TMS ) $\delta$ 14.210, 20.196, 22.674, 25.409, 29.174, $31.738,36.017,72.692,119.965,120.823,125.265,126.122,126.504,136.721$, 139.113, 144.755, 152.456, 162.111. Elem. Anal.: Calcd for $\mathrm{C}_{36} \mathrm{H}_{40} \mathrm{~N}_{2} \mathrm{O}_{4} \mathrm{~S}_{5}$ : C, 59.64; H, 5.56; N, 3.86. Found: C, 60.19; H, 5.38; N, 4.11.

\section{Electrochemical polymerization in cholesteric LC}

Chiral inducer (S)-D8Tt-B*, monomer 2,7-di(2-furyl)-fluorene (DFF) and supporting salt tetrabutylammonium perchlorate (TBAP) were dissolved in nematic LC 6CB followed by sufficient mixing for preparing the cholesteric LC electrolyte solutions. The electrolyte solution was injected between two indium-tin-oxide (ITO)-coated glass electrodes, separated by a Teflon spacer of $0.20 \mathrm{~mm}$ thickness. The polymerization cell was first heated to an isotropic phase, and then cooled and kept at room temperature. $4.0 \mathrm{~V}$ of direct current was applied to the ITO electrodes to carry out electrochemical polymerization. After 7-20 min, the conjugated polymer films P-DFF were obtained (Fig. S1, Electronic Supplementary Information). Constituents of LC electrolyte solution and polymerization conditions are listed in Table 1.

Table 1. Components of the cholesteric LC electrolyte and polymerization conditions ${ }^{\text {a }}$

\begin{tabular}{|c|c|c|c|c|}
\hline Compound & $\begin{array}{l}\text { Mass } \\
(\mathrm{mg})\end{array}$ & $\begin{array}{c}\text { Mole number } \\
(\mu \mathrm{mol})\end{array}$ & $\begin{array}{c}\text { Molarity } \\
(\%)\end{array}$ & Molecular structure \\
\hline $6 \mathrm{CB}$ & 49.6 & 188 & 97.4 & \\
\hline$(S)-\mathrm{D} 8 \mathrm{Tt}-\mathrm{B} *$ & 2.7 & 3.77 & 2.0 & \\
\hline TBAP & 0.129 & 0.38 & 0.2 & \\
\hline DFF & 0.225 & 0.78 & 0.4 & \\
\hline
\end{tabular}

\footnotetext{
a $4.0 \mathrm{~V} \mathrm{DC}$ at room temperature.
} 
Synthesis and characterization of a novel donor-acceptor-donor chiral inducer and application in electrochemical polymerization

Jiuchao Dong, Kohsuke Kawabata, and Hiromasa Goto*

J. Mater. Chem. C, 2015,3, 2024-2032.

\section{Characterization methods}

${ }^{1} \mathrm{H}$ NMR and ${ }^{13} \mathrm{C}$ NMR spectra were recorded using a JNM-ECS (JEOL, 400MHz). Chemical shifts were given in parts per million and coupling constant $(J)$ in $\mathrm{Hz}$. Elemental analysis was carried out by using Perkin-Elmer 2400 CHN Elemental Analyzer. Microscopic observations were performed under crossed Nicols using a Nikon Eclipse LV100 polarizing optical microscope (POM) equipped with a JHT TH-600PM and L-600 heating and cooling stage (Linkam). Thermodynamic properties of compounds were determined using a Seiko Instrument Exstar7000 DSC under nitrogen atmosphere at a constant heating and cooling rate of $5{ }^{\circ} \mathrm{C} / \mathrm{min}$. UV-vis absorption spectra were obtained using a JASCO V-630 UV-vis spectrophotometer. Circular dichroism (CD) and optical rotatory dispersion (ORD) were obtained using a JASCO J-720 spectrometer with an ORDE-307W ORD unit. Cyclic voltammetry (CV) data were obtained using an e-corder 201 measurement system and a PC running eDAQ software. Surface structure of the polymer film was studied by scanning electron microscopy (SEM) using a JSM-7000F (JEOL).

\section{Chemicals}

$N$-Bromosuccinimide was purchased from Nacalai Tesque (Japan). (R)-(-)-2-octanol, diisopropyl azodicarboxylate, 4,7-bis(4,4,5,5-tetramethyl-1,3,2-dioxaborolan-2yl)benzo[c][1,2,5]-thiadiazole and tetrakis(triphenylphosphine)palladium(0) were purchased from Tokyo Chemical Industry (TCI, Japan). Triphenylphosphine and sodium carbonate was obtained from Wako Pure Chemical (Japan). Synthesis of thieno[3,2-b]thiophene-2-carboxylic acid and 2,7-di(2-furyl)-fluorene have been previously reported. ${ }^{12}$

\section{Results and discussion}

\section{Inducer $(S)$-D8Tt-B*}

Thermodynamic properties. Differential scanning calorimetry (DSC) curves of $(S)$-D8Tt-B* is measured (Fig. S2, Electronic Supplementary Information), no mesophase appears during either heating or cooling. However, Schlieren texture of the corresponding solid state is observed by polarizing optical microscope (POM) (Fig. 2). This result indicates that $(S)$-D8Tt-B* exhibits nematic-like arrangement. We have reported the structural isomer of $(S)$-D8Tt-B* $\square$ D8Tt-B (Fig. S3, Electronic Supplementary Information), which has $n$-octyl groups and shows smectic A phase. ${ }^{17}$ Compared with D8Tt-B, $(S)$-D8Tt-B* possesses two chiral octyl groups in place of $n$-octyl chains. These branched structures provide considerable steric hindrance 
Synthesis and characterization of a novel donor-acceptor-donor chiral inducer and application in electrochemical polymerization

Jiuchao Dong, Kohsuke Kawabata, and Hiromasa Goto*

J. Mater. Chem. C, 2015,3, 2024-2032.

between adjacent molecules. In smectic phase, the LC molecules have stronger interaction between the lateral direction in the LC order than that between tail ends, and this feature is significant for molecules forming the smectic phase. ${ }^{21}$ Thus, the result of $(S)$-D8Tt-B* does not show smectic phase because chiral octyl groups decrease the lateral interaction of molecules, and destroy the order of the smectic phase.

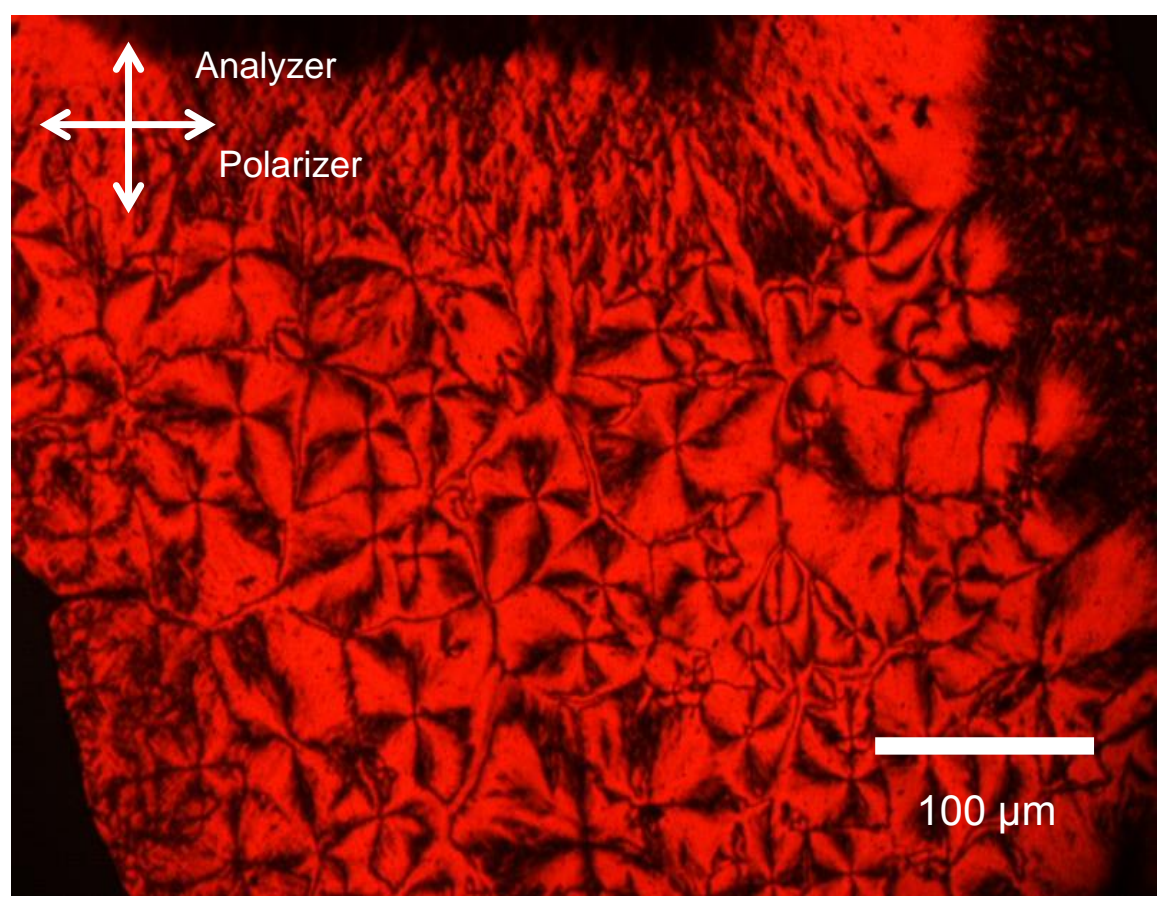

Fig. 2. POM image of $(S)$-D8Tt-B* at $90{ }^{\circ} \mathrm{C}$.

Optical properties. Optical rotatory dispersion $(\mathrm{ORD})$ of $(S)$-D8Tt-B* was measured in THF solution $(0.025 \mathrm{mM})$ (Fig. S4). The result proves that compound (S)-D8Tt-B* has positive optical rotation, contrary to the reagent $(R)-(-)$-2-octanol, due to the inversion of configuration at the chiral center in the Mitsunobu Reaction. ${ }^{22}$ Fig. 3 shows ultraviolet visible absorption (UV-vis) and circular dichroism (CD) spectra of $(S)$-D8Tt-B* in the solution state $(0.025 \mathrm{mM}$ in THF solution) and in the film (annealing and non-annealing) states. The films were fabricated by spin-coating using a dichloromethane solution of $(S)$-D8Tt-B*. The film was annealed at $130{ }^{\circ} \mathrm{C}$, then slowly cooled to $100{ }^{\circ} \mathrm{C}$ at $1{ }^{\circ} \mathrm{C} / \mathrm{min}$. In the UV-vis spectra of the solution, $(S)$-D8Tt-B ${ }^{*}$ shows two maxima in absorption at $350 \mathrm{~nm}$ and $477 \mathrm{~nm}$ that are due to $\pi-\pi^{*}$ transition of the D-A-D structure, consistent with our previous work. ${ }^{17}$ However, in the film state, the maximum absorption peaks show red-shift of about $10 \mathrm{~nm}$, due to $J$ - aggregation. ${ }^{23-25}$ After annealing, the film sample of $(S)$-D8Tt-B* shows bisignate 
Synthesis and characterization of a novel donor-acceptor-donor chiral inducer and application in electrochemical polymerization

Jiuchao Dong, Kohsuke Kawabata, and Hiromasa Goto*

J. Mater. Chem. C, 2015,3, 2024-2032.

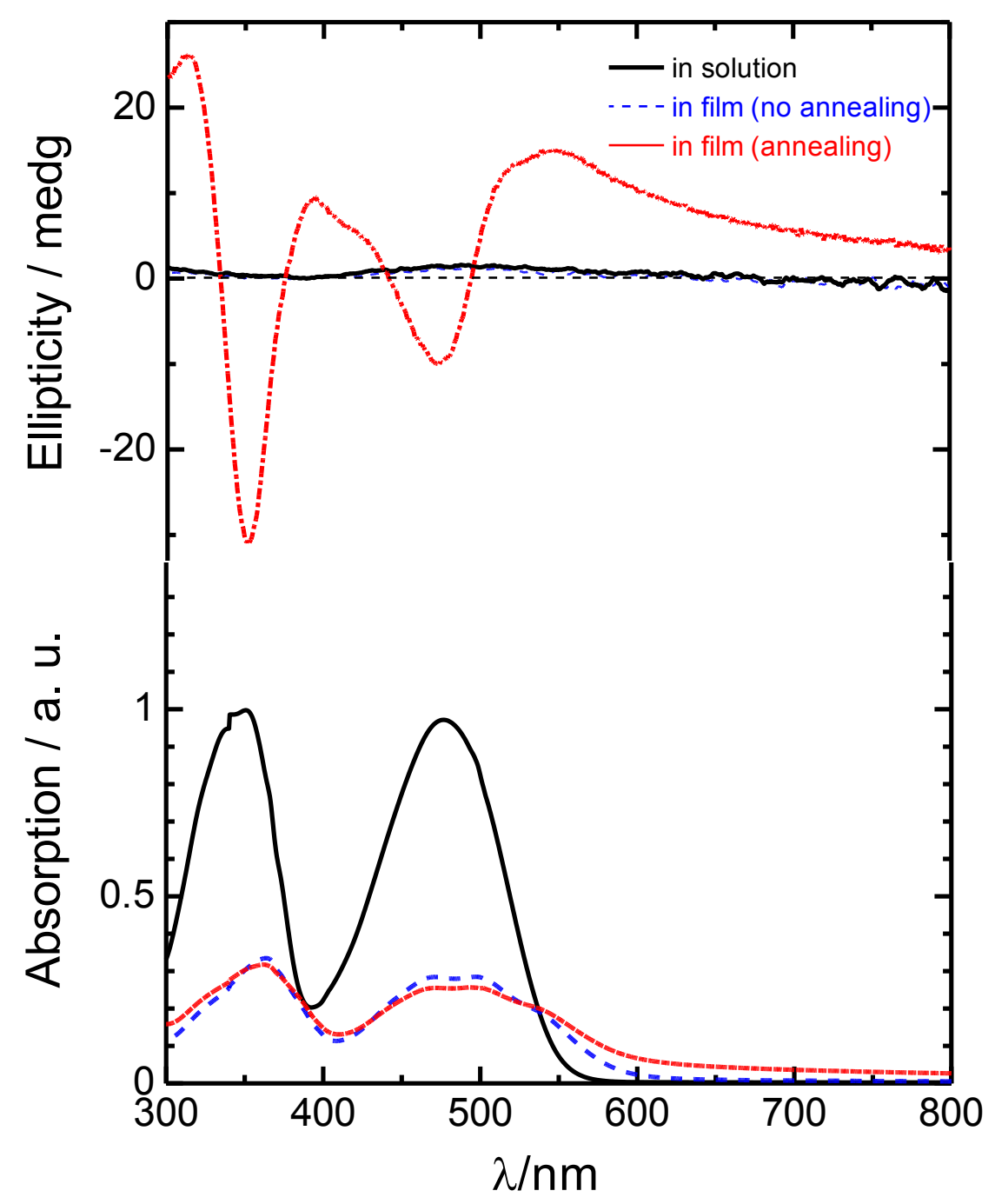

Fig. 3.CD spectra (top) and UV-vis spectra (bottom) of (S)-D8Tt-B* $(0.025 \mathrm{mM}$ in THF solution, non-annealed film state and annealed film state).

Cotton effect near its absorption maximum wavelengths in the CD spectra. CD spectra is usually used for investigating helical structure and helical arrangement of organics, because Cotton effect can indicate the asymmetric relative position between chromophores. ${ }^{26-28}$ The bisignate Cotton effect (signal is inversed between positive and negative regions) is due to Davydov splitting, it indicates that chromophores get close enough to each other so that interaction between their energy levels generate. ${ }^{12,} 29$ Therefore, the above result means that $(S)$-D8Tt-B* form chiral aggregation after annealing. This first positive and second negative Cotton effect indicates a right-handed helical aggregation. Solution-state and non-annealing film of $(S)$-D8Tt-B* show no Cotton effect, whereas annealed films show clear Cotton effect, suggesting that $(S)$-D8Tt-B* molecules rearranged and twisted by a certain angle 
Synthesis and characterization of a novel donor-acceptor-donor chiral inducer and application in electrochemical polymerization

Jiuchao Dong, Kohsuke Kawabata, and Hiromasa Goto*

J. Mater. Chem. C, 2015,3, 2024-2032.

during annealing. POM observation confirmed no fingerprint lines because the helical pitch is very short.

\section{Blend LC of $6 \mathrm{CB}$ containing $2 \mathrm{~mol} \%(S)$-D8Tt-B*}

Thermodynamic properties. Compound $(S)$-D8Tt-B* dissolves in nematic LC 6CB at $2 \mathrm{~mol} \%$. This blend LC showed cholesteric phase around room temperature. Fig. 4 shows the POM image of the fingerprint texture. The corresponding DSC curves are shown in Fig. 5A. DSC curves of 6CB are displayed in Fig. 5B as a reference. During cooling, the blend LC transform to cholesteric phase from isotropic phase at $27.1{ }^{\circ} \mathrm{C}$, and then undergoes a glass transition at $-21{ }^{\circ} \mathrm{C}$, indicating that this mixture can show stable cholesteric LC phase around room temperature during cooling. However, heating of the blend LC shows a different result; the heating curve shows exothermic peak at $-19.4{ }^{\circ} \mathrm{C}$, then two endothermic peaks at $14{ }^{\circ} \mathrm{C}$ and $27.2{ }^{\circ} \mathrm{C}$. It is worth noting that heating shows an exothermic peak. Moreover, the endothermic peak at $14{ }^{\circ} \mathrm{C}$ is similar to that of the $6 \mathrm{CB}$ (transition temperature and enthalpy change), possibly indicating a melting process. In the cooling process, the blend LC shows a glass transition at $-21{ }^{\circ} \mathrm{C}$, which is far lower than the crystallization temperature of $6 \mathrm{CB}$ (3.4 $\left.{ }^{\circ} \mathrm{C}\right)$. Furthermore, the total enthalpy change of the blend system during cooling is far less than that of $6 \mathrm{CB}$. This may be due to the $6 \mathrm{CB}$ undergoing undercooling after the addition of the chiral inducer $(S)$-D8Tt-B*. Because the undercooled glass state is metastable, when the system is disturbed by external energy, it will lose some energy to form a more stable state. Therefore, during heating, the blend system first shows an exothermic peak, indicating crystallization, and then shows an endothermic peak at 14 ${ }^{\circ} \mathrm{C}$, indicating melt.

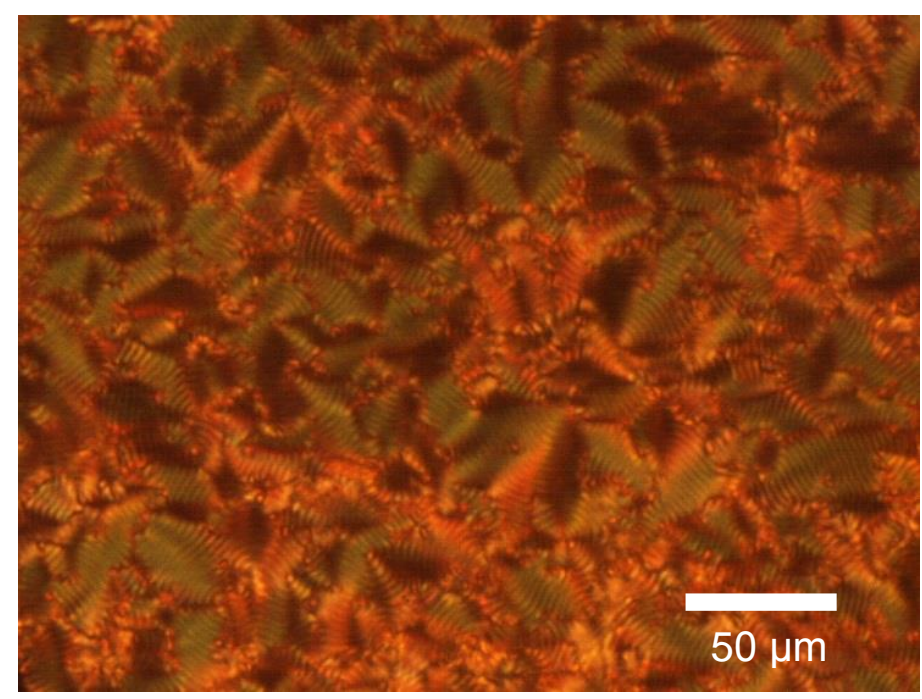

Fig. 4. POM image of fingerprint texture of blend cholesteric LC. 
Synthesis and characterization of a novel donor-acceptor-donor chiral inducer and application in electrochemical polymerization

Jiuchao Dong, Kohsuke Kawabata, and Hiromasa Goto*

J. Mater. Chem. C, 2015,3, 2024-2032.
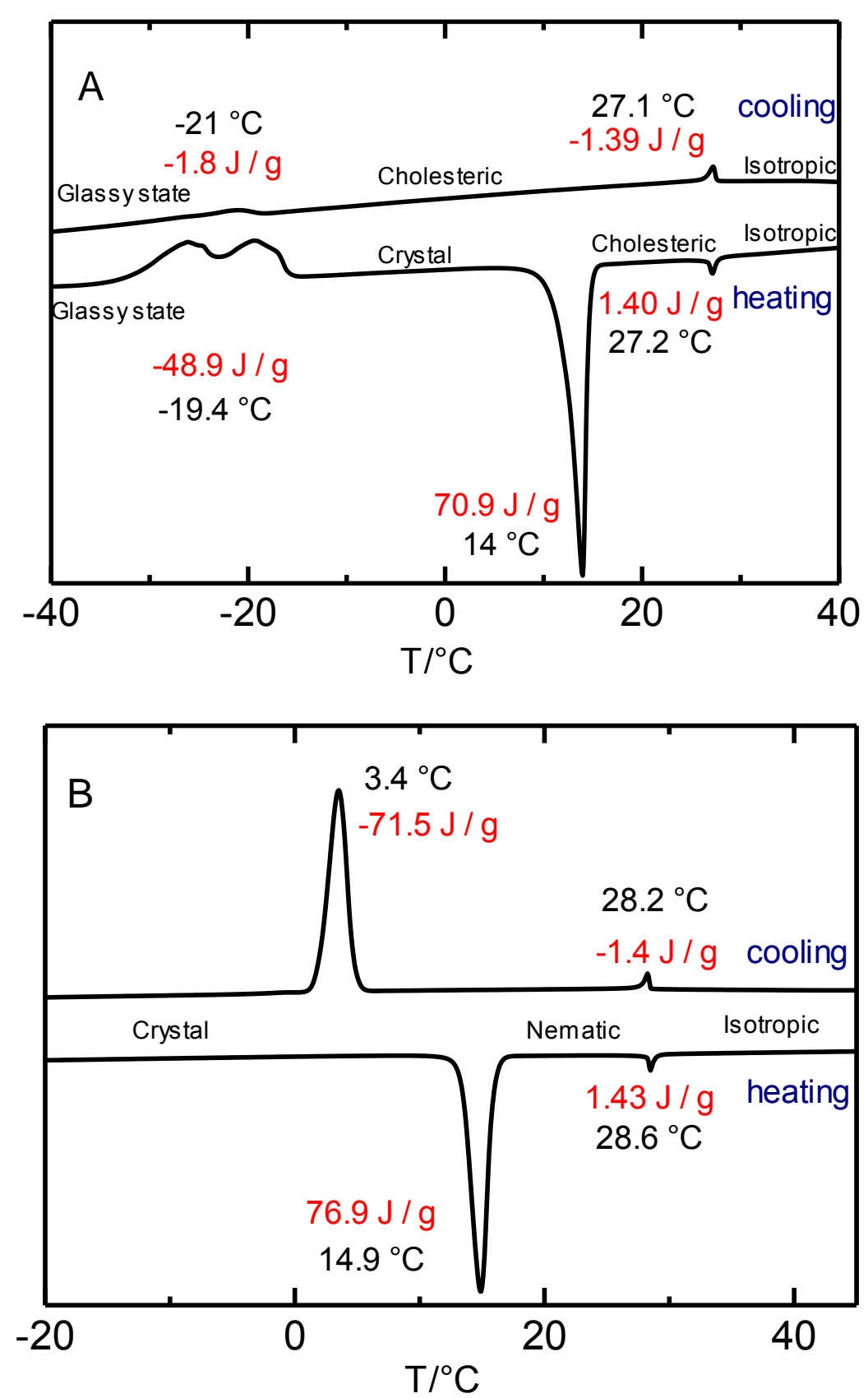

Fig. 5. DSC curves of blend cholesteric LC (A) and nematic LC 6CB (B) at a scan rate of $5{ }^{\circ} \mathrm{C} / \mathrm{min}$.

Helical sense and helical twisting power. For determination of the helical sense of $(S)$-D8Tt-B*, miscibility testing was performed. ${ }^{13}(S)$-D8Tt-B* was dissolved in 6CB 2 mol\%, and compared with standard cholesteric LC cholesteryl oleyl carbonate that possesses a left-handed helical architecture. Fig. 6A shows the POM image of the miscibility test on a glass cell at room temperature. A smectic phase was observed on 
Synthesis and characterization of a novel donor-acceptor-donor chiral inducer and application in electrochemical polymerization

Jiuchao Dong, Kohsuke Kawabata, and Hiromasa Goto*

J. Mater. Chem. C, 2015,3, 2024-2032.

the boundary of the sample and the cholesteryl oleyl carbonate. After heating to $28{ }^{\circ} \mathrm{C}$, the smectic region transfers to cholesteric phase (Fig. 6B). This means that the smectic

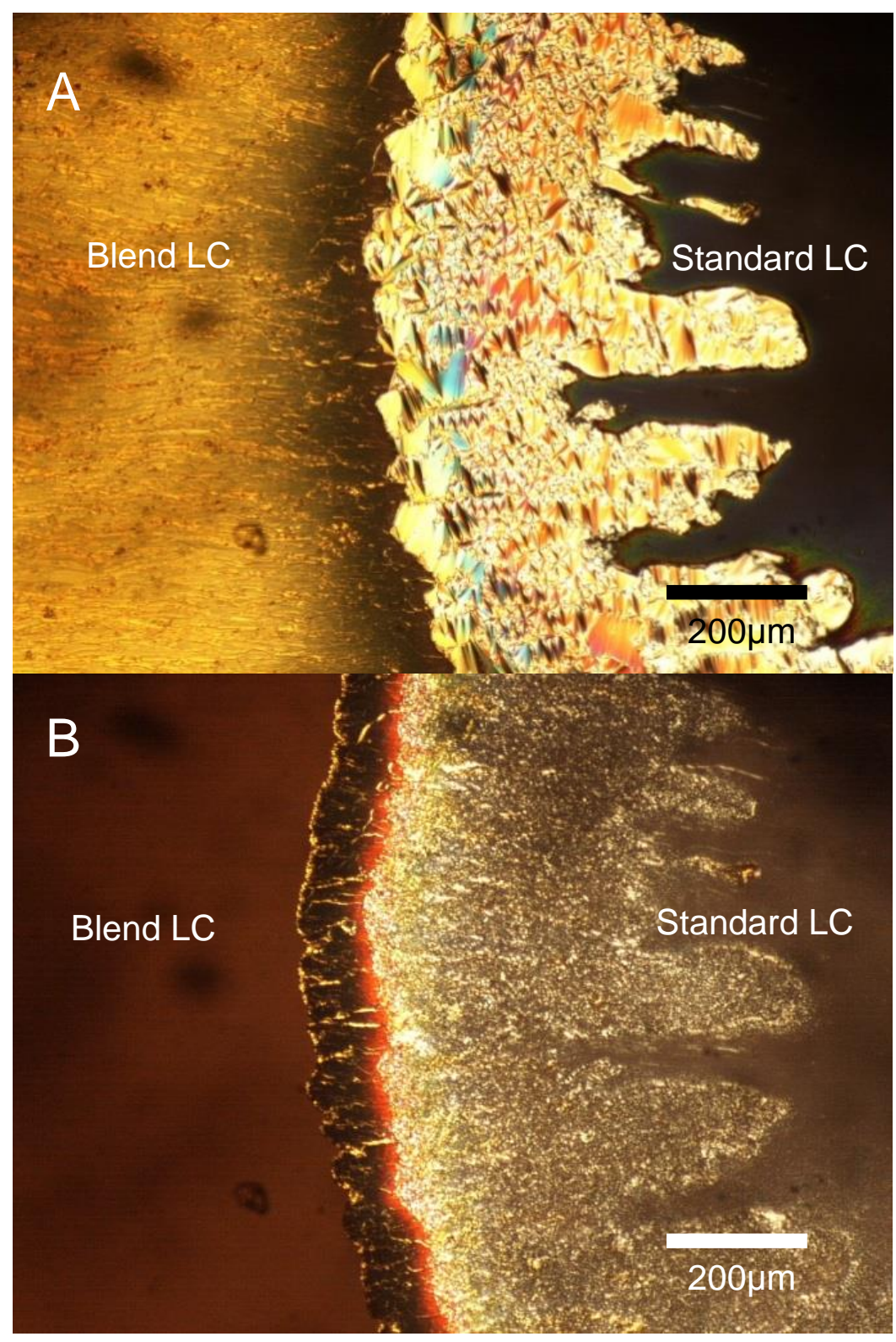

Fig. 6.POM images of miscibility test (A: room temperature, B: $28{ }^{\circ} \mathrm{C}$ ).

phase can be defined as a chiral smectic phase. If the sample and the standard LC have the opposite helical sense, the helical twist of the cholesteric LC is unwound. The boundary would show nematic phase with no chirality. However no Schlieren texture was observed in this miscibility test, indicating that the sample forms a left-handed helical architecture at the molecular level. In general, when a chiral alkyl is linked to the ester group in a chiral inducer, and its chiral center is in odd position of the chiral alkyl, the chiral inducer will be more likely to induce left-handed helical architecture in 
Synthesis and characterization of a novel donor-acceptor-donor chiral inducer and application in electrochemical polymerization

Jiuchao Dong, Kohsuke Kawabata, and Hiromasa Goto*

J. Mater. Chem. C, 2015,3, 2024-2032.

host LC. ${ }^{30}(S)$-D8Tt-B* itself possesses right-handed helical architecture, but cholesteric LC with $(S)$-D8Tt-B* forms left-handed helical architecture in 6CB. It corresponds to the ORD measurement (Fig. S5). The blend liquid shows negative optical rotation; on the other hand, $(S)-\mathrm{D} 8 \mathrm{Tt}-\mathrm{B}^{*}$ shows positive optical rotation. This result is deemed to be concerned with different interactions between inducer-inducer and inducer-host LC.

The macroscopic helical twisting power $\left(\beta_{M}\right)$ was measured by the Grandjean-Cano wedge method (Fig. S6). ${ }^{31,32}$ The $\beta_{\mathrm{M}}$ value was calculated by:

$$
\beta_{\mathrm{M}}=\left(\mathrm{p} \cdot \mathrm{c} \cdot \mathrm{M}_{\mathrm{h}} / \mathrm{M}_{\mathrm{d}}\right)^{-1}
$$

where $\mathrm{p}$ is a helical pitch of the mixture, $\mathrm{c}$ is the concentration (in wt $\%$ ) of the chiral inducer, $M_{h}$ is the molecular weight of the solvent and $M_{d}$ is the molecular weight of the chiral inducer. The result is $17.2 \mu \mathrm{m}^{-1}$, indicating that $(S)-\mathrm{D} 8 \mathrm{Tt}-\mathrm{B}^{*}$ has high helical twisting power. ${ }^{13}$

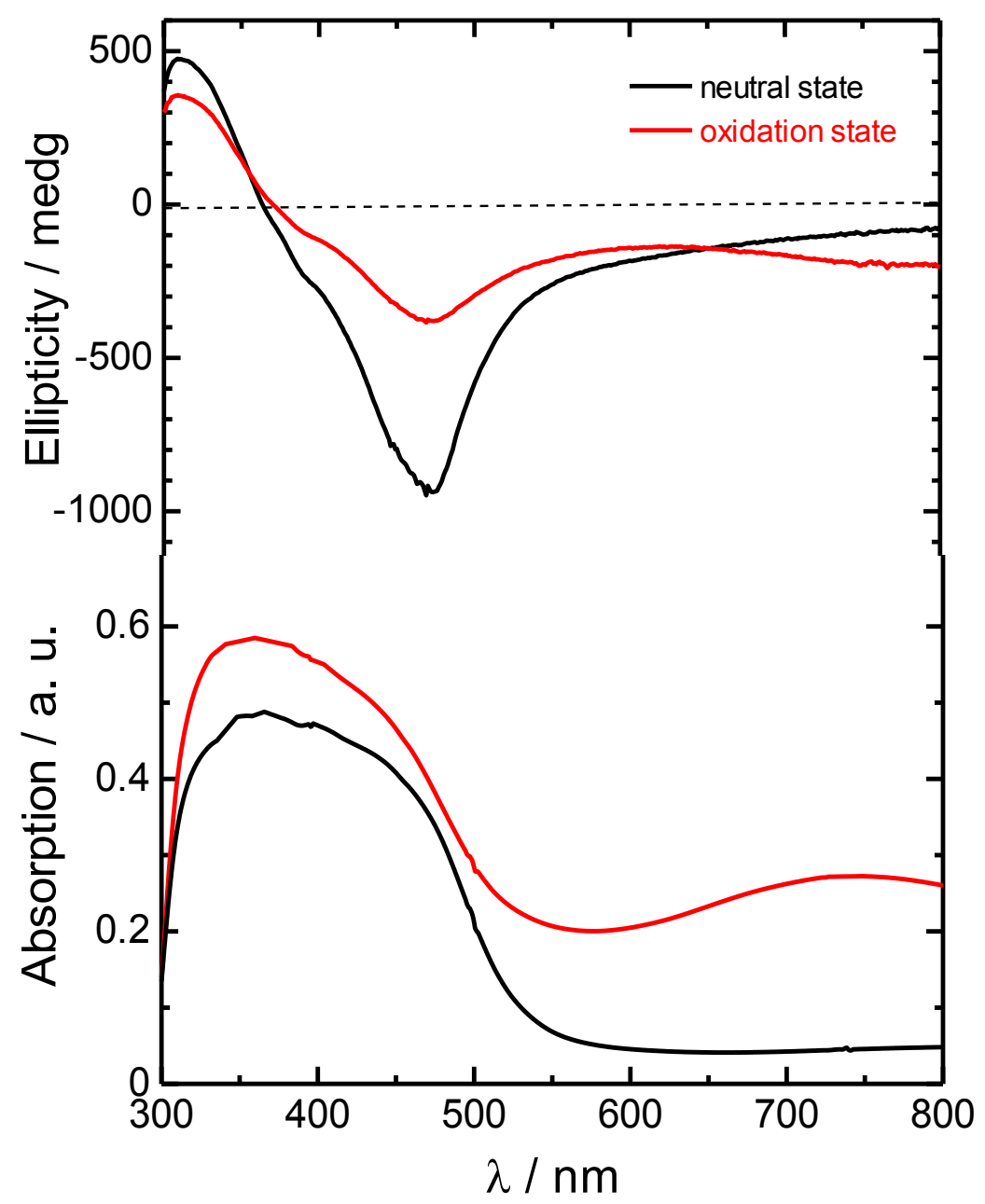

Fig. 7. CD spectra (top) and UV-vis spectra (bottom) of P-DFF in neutral state and oxidized state (polymerization time: $7 \mathrm{~min}$ ). 
Synthesis and characterization of a novel donor-acceptor-donor chiral inducer and application in electrochemical polymerization

Jiuchao Dong, Kohsuke Kawabata, and Hiromasa Goto*

J. Mater. Chem. C, 2015,3, 2024-2032.

\section{Electrochemical polymerization}

Optical properties and redox properties. Due to electrochemical doping, the pristine polymer film obtained in electrochemical polymerization is often the oxidized state. It can be reduced by using a reductive agent such as hydrazine to obtain the neutral state. We conducted an electrochemical polymerization with the 2 mol\% chiral inducer for 7 minutes. Optical properties of the resultant film were determined by UV-vis spectra and CD spectra in both oxidation and neutral states (Fig. 7). In the UV-vis spectra both oxidation and neutral states of the film exhibit absorption bands at 300-500 nm. These absorption bands can be attributed to $\pi-\pi^{*}$ transitions of the conjugated backbone. The oxidized state shows another absorption band at 600-800 nm., attributed to polarons generated by electrochemical doping. The oxidized state showed different colors than the neutral state (Fig. S7).

In the $\mathrm{CD}$ spectra, both oxidized and neutral states of the polymer show bisignate Cotton effect at their absorption maximum wavelengths. This means Davydov splitting and suggests that the chromophores (polymer backbones) form a chiral aggregation. This first negative and second positive Cotton effect indicate a left-handed helical aggregation. There is left-handed helical aggregation between polymer backbones, and this result is consistent with that of blend LC electrolyte that has left-handed helical order. It suggests that polymer backbone grow as the arrangement of LC. At 650-800 $\mathrm{nm}$, the oxidized state film shows intense signal compared with the neutral state. The signal is due to the polaron band in the UV-vis.

Surface texture. POM and scanning electron microscope (SEM) images of the polymers are shown in Fig. 8. The polymer films display a spiral texture quite similar to the fingerprint texture of cholesteric, hometropic, LC. This demonstrated that the polymer transcribed the macroscopic arrangement of the cholesteric LC during the electrochemical polymerization. The periodic convex-concave structure reveals that an epitaxial electropolymerization depends on molecular alignment of the matrix LC. Fig. 9 shows the polymer surfaces (A: polymerization time $=10 \mathrm{~min}, 2 \mathrm{~mol} \%$ chiral inducer; B: $20 \mathrm{~min}, 2 \mathrm{~mol} \%$ chiral inducer; $10 \mathrm{~min}, 4 \mathrm{~mol} \%$ chiral inducer). Note that increase of polymerization time resulted in highly developed convex-concave structure. Increase of the amount of chiral inducer resulted in a decrease of distance between convex structures (changed from ca. $1 \mu \mathrm{m}$ to ca. $0.5 \mu \mathrm{m}$ ). These results are also due to that polymer backbone grow as the arrangement of LC. Because cholesteric LC has helical architecture, the growth direction of polymer backbones periodically changes(the period is consistent with the helical pitch of cholesteric LC) and shows anisotropy, 
Synthesis and characterization of a novel donor-acceptor-donor chiral inducer and application in electrochemical polymerization

Jiuchao Dong, Kohsuke Kawabata, and Hiromasa Goto*

J. Mater. Chem. C, 2015,3, 2024-2032.

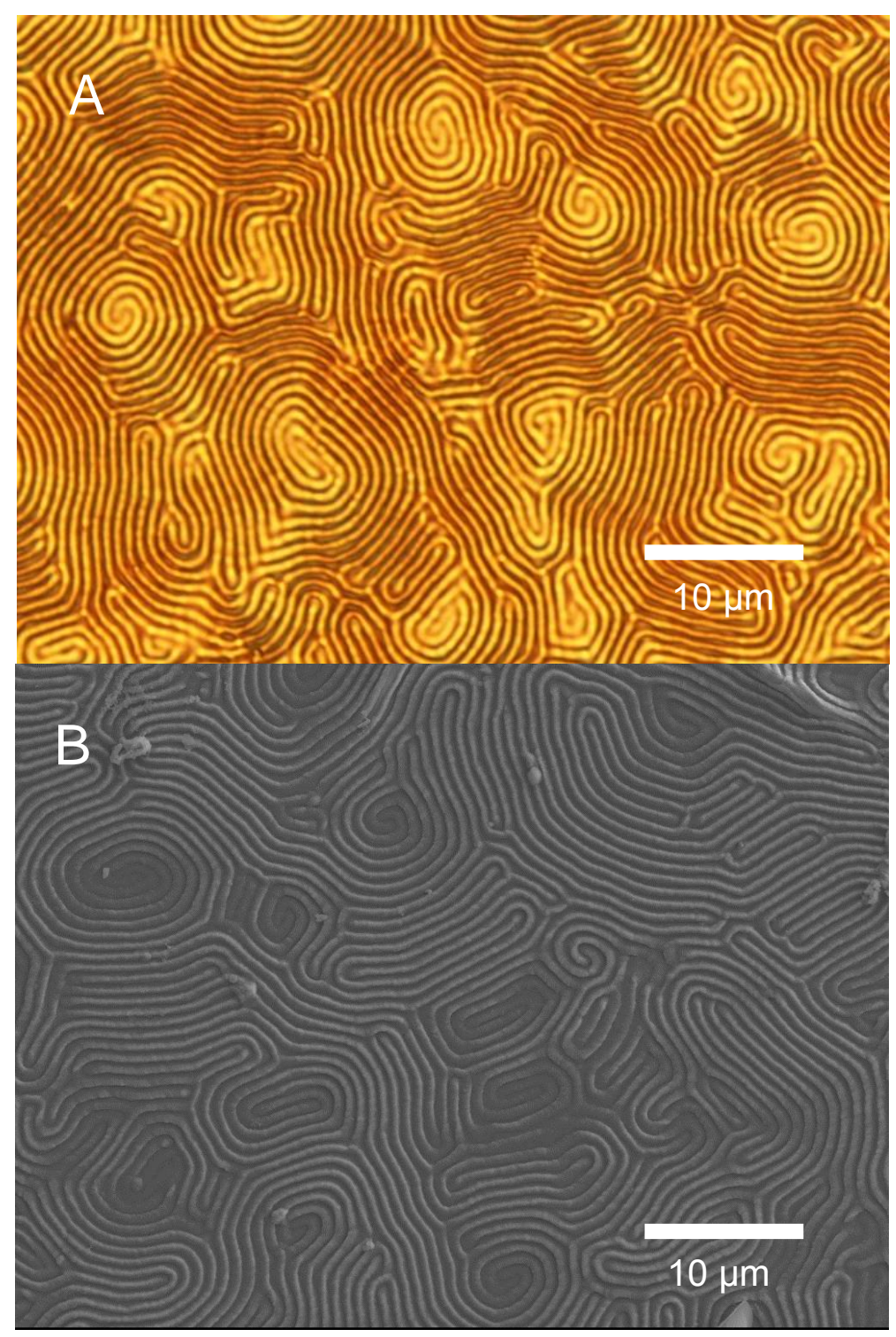

Fig. 8. POM image (A) and SEM image (B) of P-DFF films (inducer concentration: $2 \%$ molar; polymerization time: $20 \mathrm{~min}$ ).

which results the convex-concave structure. Therefore, when polymerization time increase, increased length of polymer backbones make the degree of anisotropy increase, then the convex-concave structure highly develops. For the same principle, when amount of chiral inducer increase, the helical pitch of cholesteric LC decreases that makes the helical period of polymer backbones decrease, then distance between convex structures (about a half of helical period) decreases. It suggest that the surface structure of the films depends on polymerization time and inducer concentration.

The color of the neutral state polymer film is yellow. Irradiation of white light at different incident angles results in changes in the reflected light, such as jewel beetle-like iridescent (Fig. 10). We determined the colors of reflection spectra upon 
Synthesis and characterization of a novel donor-acceptor-donor chiral inducer and application in electrochemical polymerization

Jiuchao Dong, Kohsuke Kawabata, and Hiromasa Goto*

J. Mater. Chem. C, 2015,3, 2024-2032.

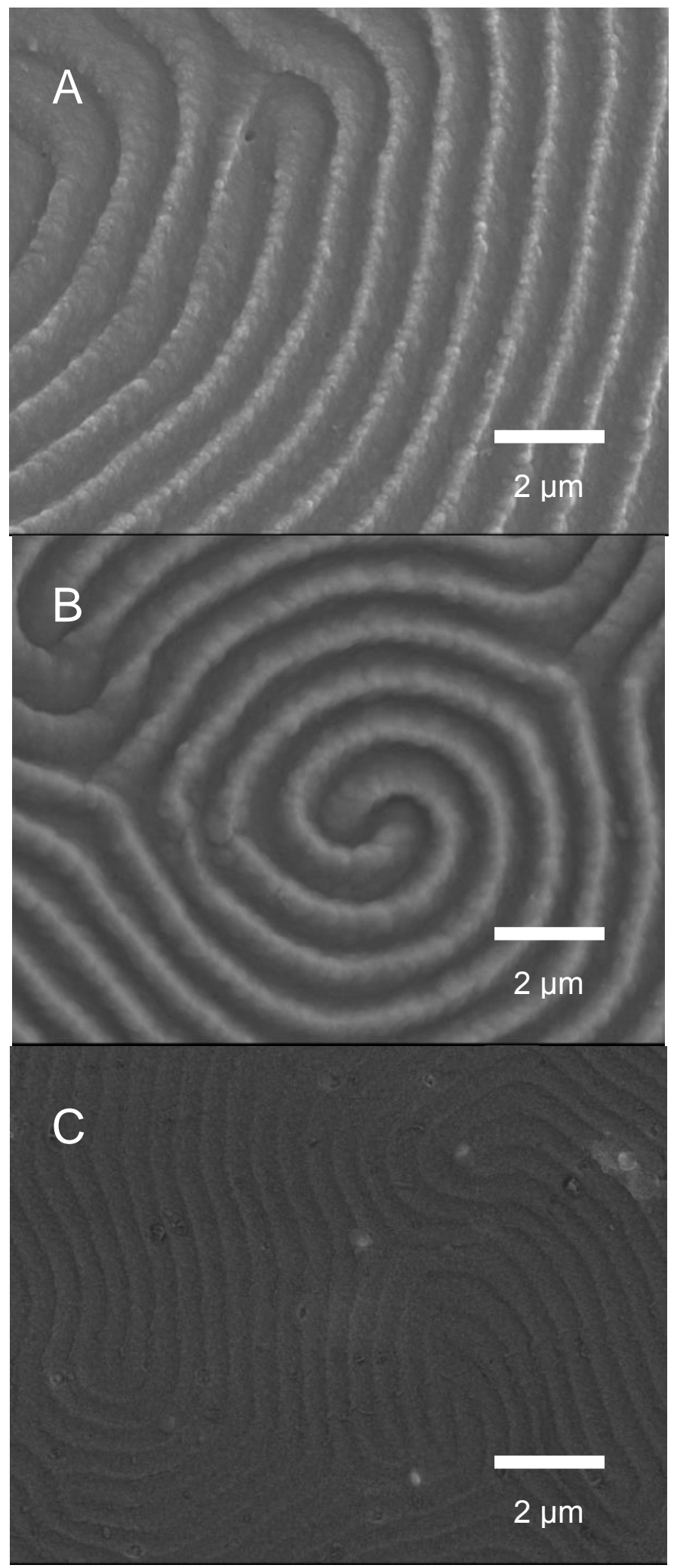

Fig. 9. SEM images of P-DFF films obtained with different polymerization conditions (A: $10 \mathrm{~min}, 2 \mathrm{~mol} \%$ chiral inducer; B: $20 \mathrm{~min}, 2 \mathrm{~mol} \%$ chiral inducer; C: $10 \mathrm{~min}, 4 \mathrm{~mol} \%$ chiral inducer). 
Synthesis and characterization of a novel donor-acceptor-donor chiral inducer and application in electrochemical polymerization

Jiuchao Dong, Kohsuke Kawabata, and Hiromasa Goto*

J. Mater. Chem. C, 2015,3, 2024-2032.

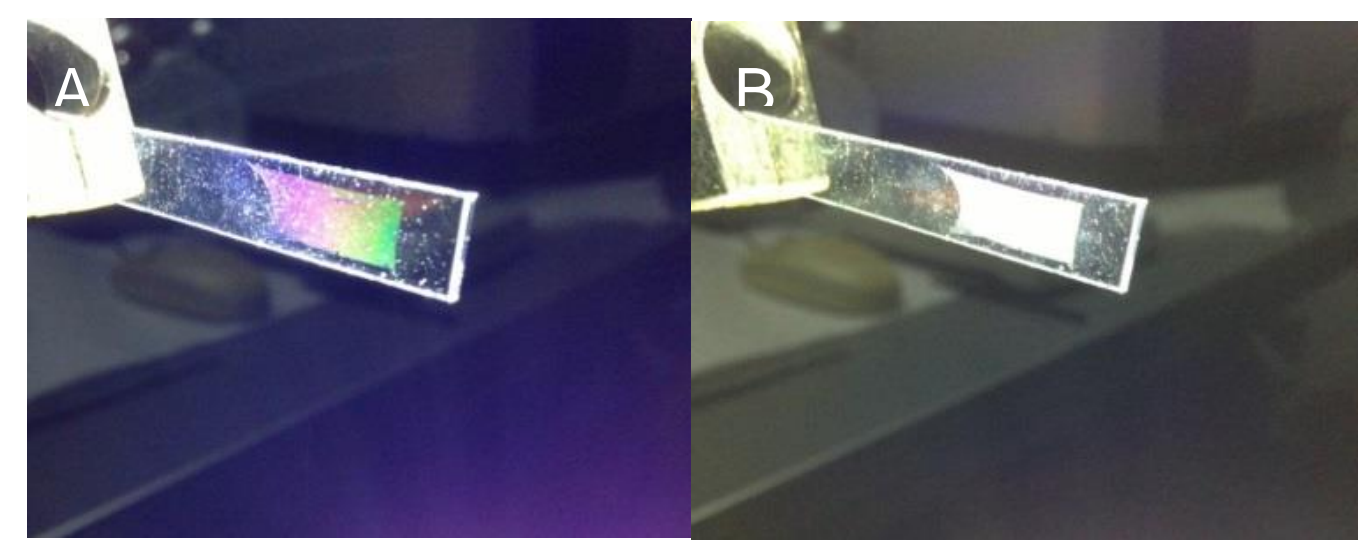

Fig. 10. Images of P-DFF films showing rainbow (A) and white (B) reflected light.
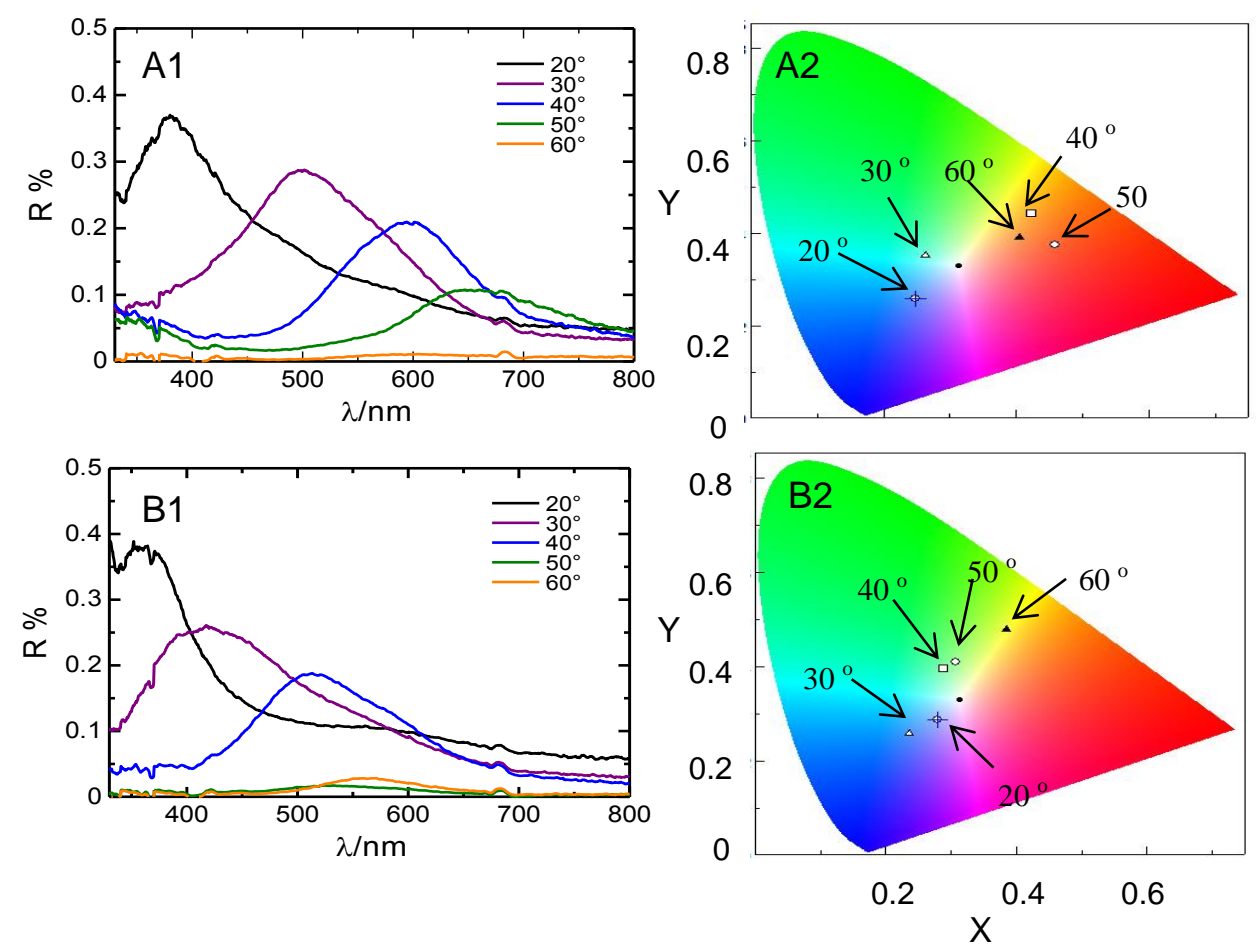

Fig. 11.Reflection spectra (1) and CIE color-space chromaticity diagram (2) of the polymer film with different fixed incident angles and changed detection angles. A: incident angle $20^{\circ}$; B: $30^{\circ}$.

different incident angles and detection angles, then transformed the data to the International Commission on Illumination (CIE) color-space chromaticity diagram. The results are summarized in Fig. 11. In this experiment, incident angle was defined as this: the angle between incident light and normal of sample surface; detection angle was defined as Fig. S8. When incident angle was fixed at $20^{\circ}$, increase of detection angle resulted in red-shift and decrease of the intensity. When incident angle increased to $30^{\circ}$, reflection band of each detection angles gradually blue-shifted, and the 
Synthesis and characterization of a novel donor-acceptor-donor chiral inducer and application in electrochemical polymerization

Jiuchao Dong, Kohsuke Kawabata, and Hiromasa Goto*

J. Mater. Chem. C, 2015,3, 2024-2032.

intensities slightly decreased. These results are due to the diffraction derived from the periodic convex-concave strips of film surface structure. The scales of these convex-concave strips are comparable to the light wavelength. When light irradiates the convex-concave strips, different positions of incidence result in different reflection paths (Fig. S9); different reflection paths generate optical path differences, resulting in diffraction. Because optical path difference is related to both incident and detection angles, different reflected light is observed at different angles.

We also carried out irradiation with green laser light with vertical incidence (532 $\mathrm{nm}$, Fig. S10). The diffraction rings appeared due to random grating function. These result reveals that the periodic convex-concave strips are sufficiently compact to generate diffraction.

Electrochemical properties. Electrochemical properties of the polymer films (prepared with LC electrolyte solution with $(S)$-D8Tt-B* in 2 mol\%; polymerization time $=10 \mathrm{~min}$ ) were examined with a cyclic voltammetry $(\mathrm{CV})$ at scan rates of 10 , $20,40,60,80$ and $100 \mathrm{mV} / \mathrm{sin} 0.1 \mathrm{M}$ TBAP/acetonitrile solution (Fig. 12). The electrochemical cell contains an ITO working electrode coated by the polymer sample, a platinum wire counter electrode, and an $\mathrm{Ag} / \mathrm{AgNO}_{3}$ reference electrode. The redox curves reveal a quasi-reversible redox process with relatively low oxidation potential. The current value increased gradually with the increase of scan rates. These results suggest that the electron transfer can be accessed by applied voltage. Under all conditions the polymer adhered well to the electrode.

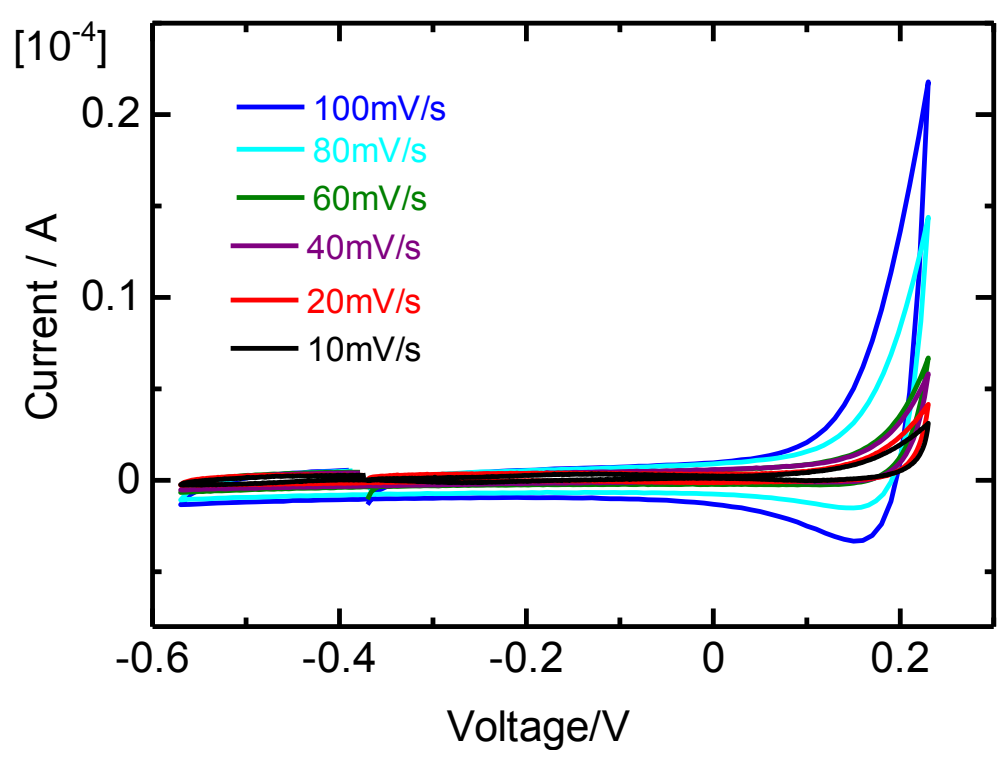

Fig. 12. CV curves of P-DFF film. 
Synthesis and characterization of a novel donor-acceptor-donor chiral inducer and application in electrochemical polymerization

Jiuchao Dong, Kohsuke Kawabata, and Hiromasa Goto*

J. Mater. Chem. C, 2015,3, 2024-2032.

\section{Conclusions}

In this work, a novel D-A-D structure chiral inducer (S)-D8Tt-B* was synthesized. This chiral inducer showed Schlieren texture and right-handed helical aggregation in the glassy state. However, it induced left-handed helical architecture in 6CB with a macroscopic helical twisting power, $\beta_{\mathrm{M}}=17.2 \mu \mathrm{m}^{-1}$. The mixture showed stable cholesteric LC phase at room temperature. The conjugated polymer films obtained in the cholesteric LC electrolyte solution showed circular dichroism with negative Cotton effect, indicating left-handed helical aggregation. The conjugated polymer films thus transcribed the fingerprint texture of the cholesteric LC electrolyte solution with periodic concave-convex surface structure. The periodical structure showed selective reflection of light. Concentration of chiral inducer and polymerization time quantitatively affected the surface structure of the polymer films.

\section{Acknowledgements}

We would like to thank the OPEN FACILITY, Research Facility Center for Science and Technology, University of Tsukuba, for allowing us to use the nuclear magnetic resonance spectrometer and differential scanning calorimetry instrument. We would like to thank the National Institute for Materials Science for the use of the scanning electron microscope. We would also like to thank Tomoaki Joh, Hirotsugu Kawashima and Aohan Wang for their help and support.

\section{Notes and references}

1 X. Guo, M. Zhang, L. Huo, F. Xu, Y. Wu and J. Hou, J. Mater. Chem., 2012, 22, $21024-$ 2103.

2 Y. Huang, X. Guo, F. Liu, L. Huo, Y. Chen, T. P. Russell, C. C. Han, Y. Li, and J. Hou, Adv. Mater., 2012, 24, 3383-3389.

3 M. Horie, L. A. Majewski, M. J. Fearn, C-Y. Yu, Y. Luo, A. Song, B. R. Saunders and M.

L. Turner, J. Mater. Chem., 2010, 20, 4347-4355.

4 H. Tanaka, S. Watanabe, H. Ito, K. Marumoto, and S. Kuroda, Appl. Phys. Lett., 2009, 94, 103308.

5 Y. Honsho, A. Saeki and S. Seki, International Journal of Spectroscopy, 2012, 2012, 7.

6 J. Kulhánek and F. Bureš, Beilstein J. Org. Chem., 2012, 8, 25-49.

7 R. Karpicz, S. Puzinas, S. Krotkus, K. Kazlauskas, S. Jursenas, J. V. Grazulevicius, S. Grigalevicius and V. Gulbinas, J. Chem. Phys., 2011, 134, 204508.

8 Y. Kunugi, I. Tabakovic, A. Canavesi and L. L. Miller, Synth. Met., 1997, 89, 227. 
Synthesis and characterization of a novel donor-acceptor-donor chiral inducer and application in electrochemical polymerization

Jiuchao Dong, Kohsuke Kawabata, and Hiromasa Goto*

J. Mater. Chem. C, 2015,3, 2024-2032.

9 Y. Kunugi, K. R. Mann, L. L. Miller and C. L. Exstrom, J. Am. Chem. Soc., 1998, 120, 589.

10 A. Tsumua, H. Koezuka and T. Ando, Appl. Phys. Lett., 1986, 49, 1210.

11 K. Kawabata, S. Nimori and H. Goto, ACS Macro Lett., 2013, 2, 587-591.

12 K. Kawabata, M. Takeguchi and H. Goto, Macromolecules, 2013, 46, 2078-2091.

13 H. Hayashi, A. Wang, K. Kawabata, H. Goto, Mater. Chem. Phys., 2013, 137, 816-824.

14 H. Goto, RSC. Adv., 2013, 3, 6347-6355.

15 H. Yoneyama, A. Tsujimoto and H. Goto, Macromolecules, 2007, 40, 5279-5283.

16 A. Wang, K. Kawabata, H. Kawashima and H. Goto, Polymer, 2013, 54, 3821-3827.

17 J. Dong, K. Kawabata, T. Seino, F. Yang, and H. Goto, LiqCryst., 2013, 40, 1455-1465.

18 N. S. Baek, S. K. Hau, H. L. Yip, O. Acton, K. S. Chen and A. K. Y. Jen, Chem. Mater, 2008, 20, 5734-5736.

19 D. Deng, Y. Yang, J. Zhang, C. He, M. Zhang, Z. G. Zhang, Z. Zhang and Y. Li, Org. Electron., 2011, 12, 614-622.

20 J. M. Raimundo, P. Blanchard, H, Brisset, S, Akoudad and J. Roncali, Chem. Commun., 2000, 939-940.

21 S. Matsumoto, and I. Tsunoda, Ekisyo no saishingijutsu [Latest technologies of liquid crystal], Kogyo Chosakai, Tokyo, 1983.

22 R. Dembinski, Eur. J. Org. Chem., 2004, 2763-2772.

23 J. M. Kuiper and J. B. F. N. Engberts, Langmuir, 2004, 20, 1152-1160.

24 H. Tachibana, F. Sato, S. Terrettaz, R. Azumi, T. Nakamura, H. Sakai, M. Abe and M. Matsumoto, Thin Solid Films, 1998, 813-815.

25 J. R. Lenhard and B. R. Hein, J. Phys. Chem., 1996, 100, 17287-17296.

26 T. Yamamoto, T. Adachi, and M. Suginome, ACS Macro Lett., 2013, 2, 790-793.

27 K. Kishikawa, T. Sugiyama, T. Watanabe, S. Aoyagi, M. Kohri, T. Taniguchi, M. Takahashi, and S. Kohmoto, J. Phys. Chem. B, 2014, 118, 10319-10332.

28 Y. Nagata, T. Nishikawa and M. Suginome, Chem. Commun., 2012, 48, 11193-11195.

29 N. Berova, K. Nakanishi, and R. W. Wood, Circular Dichroism: Principles and Applications, Wiley, New York, 2nd ed., 2000.

30 Editorial Committee of liquid crystal manual, Ekisyobenran [Liquid crystal manual], Maruzen Publishing, Tokyo, 2000.

31 C. Blanc, N. Zuodar, I. Lelidis, M. Kleman and J. L. Martin, Phys. Rev. E, 2004, 69, 011705.

32 J. F. Strömer, D. Marenduzzo, C. V. Brown, J. M. Yeomans and E. P. Raynes, J. Appl. Phys., 2006, 99, 064911. 
Synthesis and characterization of a novel donor-acceptor-donor chiral inducer and application in electrochemical polymerization

Jiuchao Dong, Kohsuke Kawabata, and Hiromasa Goto*

J. Mater. Chem. C, 2015,3, 2024-2032.

Supplementary information for:

\section{Synthesis and characterization of a novel donor-acceptor-donor chiral inducer and application in electrochemical polymerization}

Jiuchao Dong, Kohsuke Kawabata, and Hiromasa Goto*

Division of Materials Science, Faculty of Pure and Applied Sciences,

University of Tsukuba, Tsukuba, Ibaraki 305-8573, Japan

Correspondence to H. Goto, Tel: +81-298-53-5128, fax: +81-298-53-4490

Email: gotoh@ims.tsukuba.ac.jp 
Synthesis and characterization of a novel donor-acceptor-donor chiral inducer and application in electrochemical polymerization

Jiuchao Dong, Kohsuke Kawabata, and Hiromasa Goto*

J. Mater. Chem. C, 2015,3, 2024-2032.

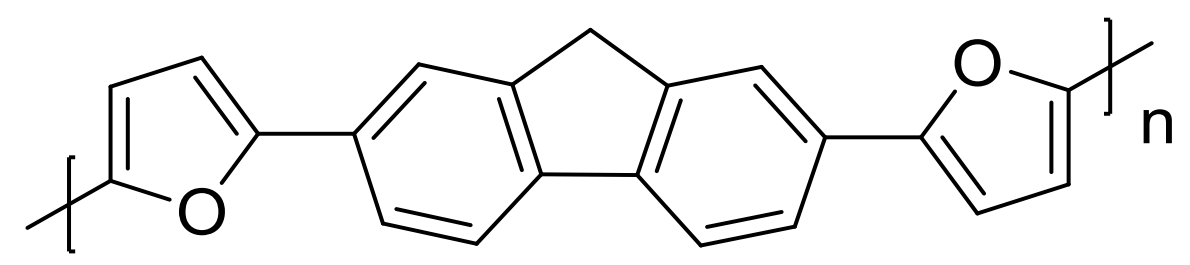

Fig. S1. Conjugated polymer P-DFF.

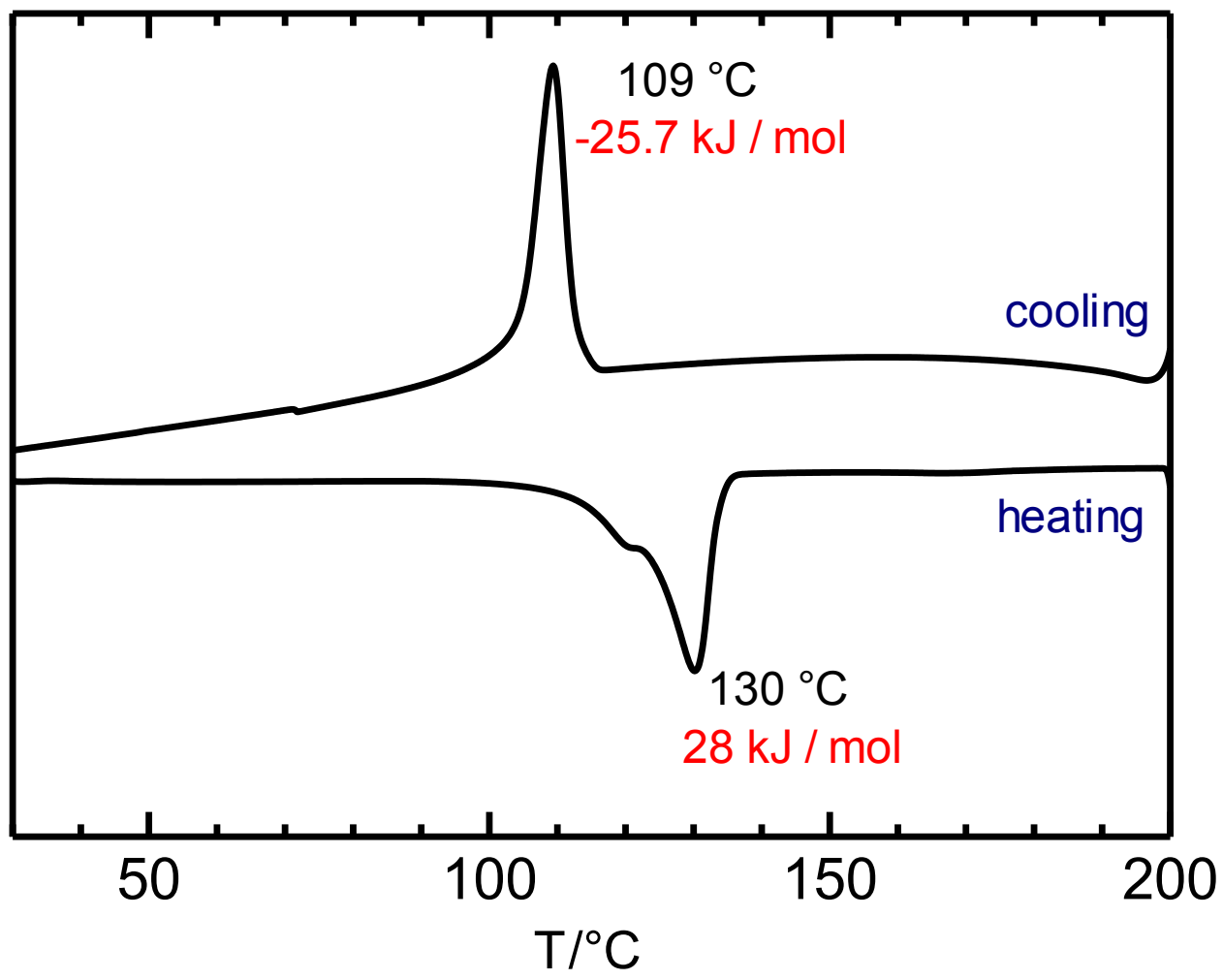

Fig. S2. DSC curves of compound (S)-D8Tt-B* at a scan rate of $5^{\circ} \mathrm{C} / \mathrm{min}$. 
Synthesis and characterization of a novel donor-acceptor-donor chiral inducer and application in electrochemical polymerization

Jiuchao Dong, Kohsuke Kawabata, and Hiromasa Goto*

J. Mater. Chem. C, 2015,3, 2024-2032.

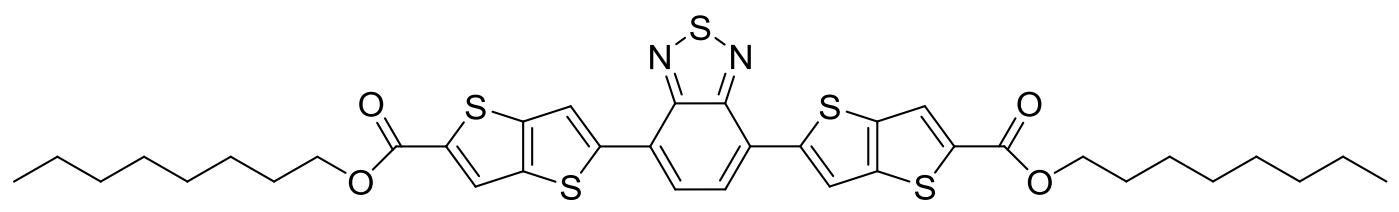

Fig. S3. Compound D8Tt-B.

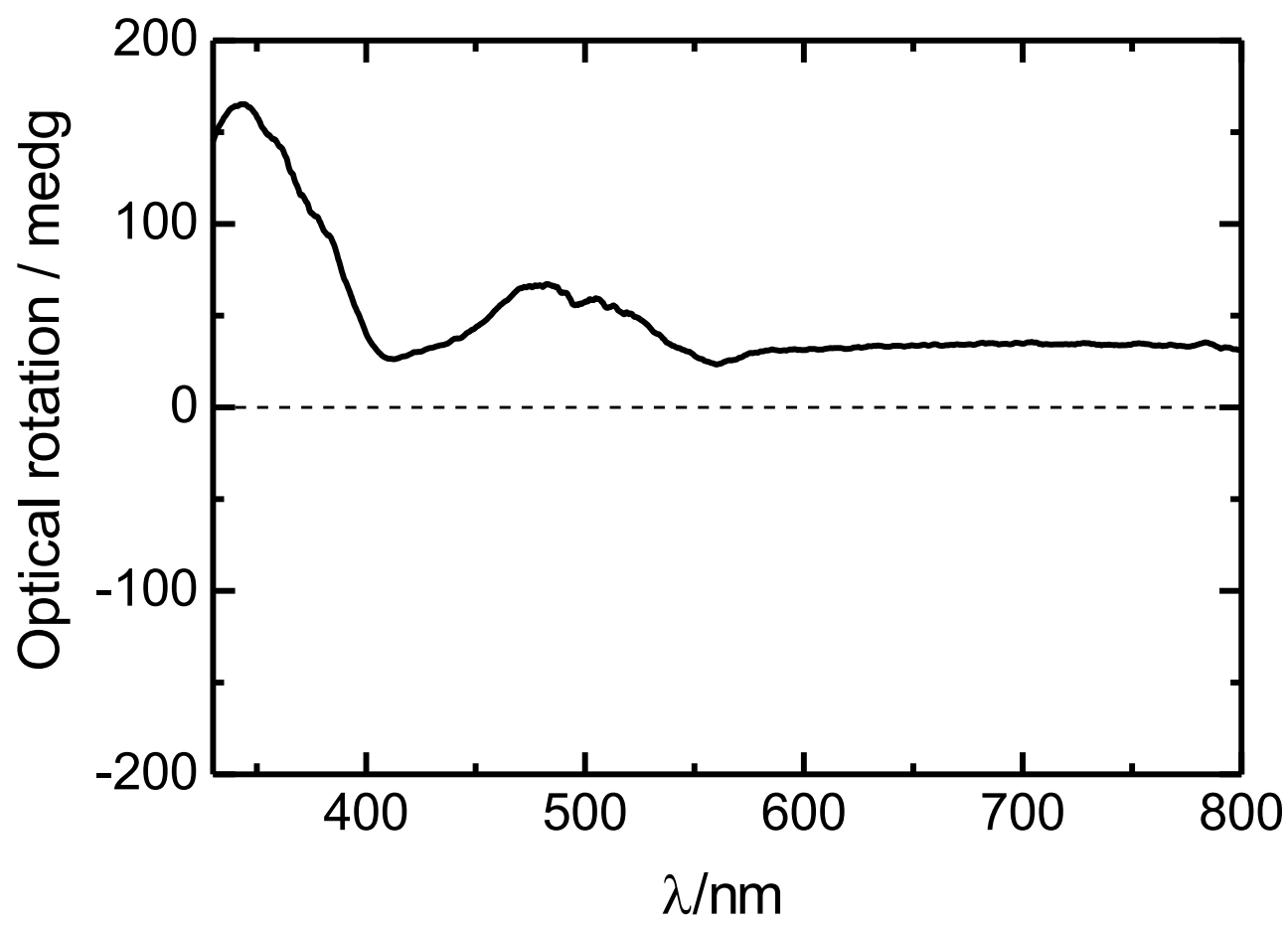

Fig. S4. ORD of compound (S)-D8Tt-B* (0.025 mM in THF solution). 
Synthesis and characterization of a novel donor-acceptor-donor chiral inducer and application in electrochemical polymerization

Jiuchao Dong, Kohsuke Kawabata, and Hiromasa Goto*

J. Mater. Chem. C, 2015,3, 2024-2032.

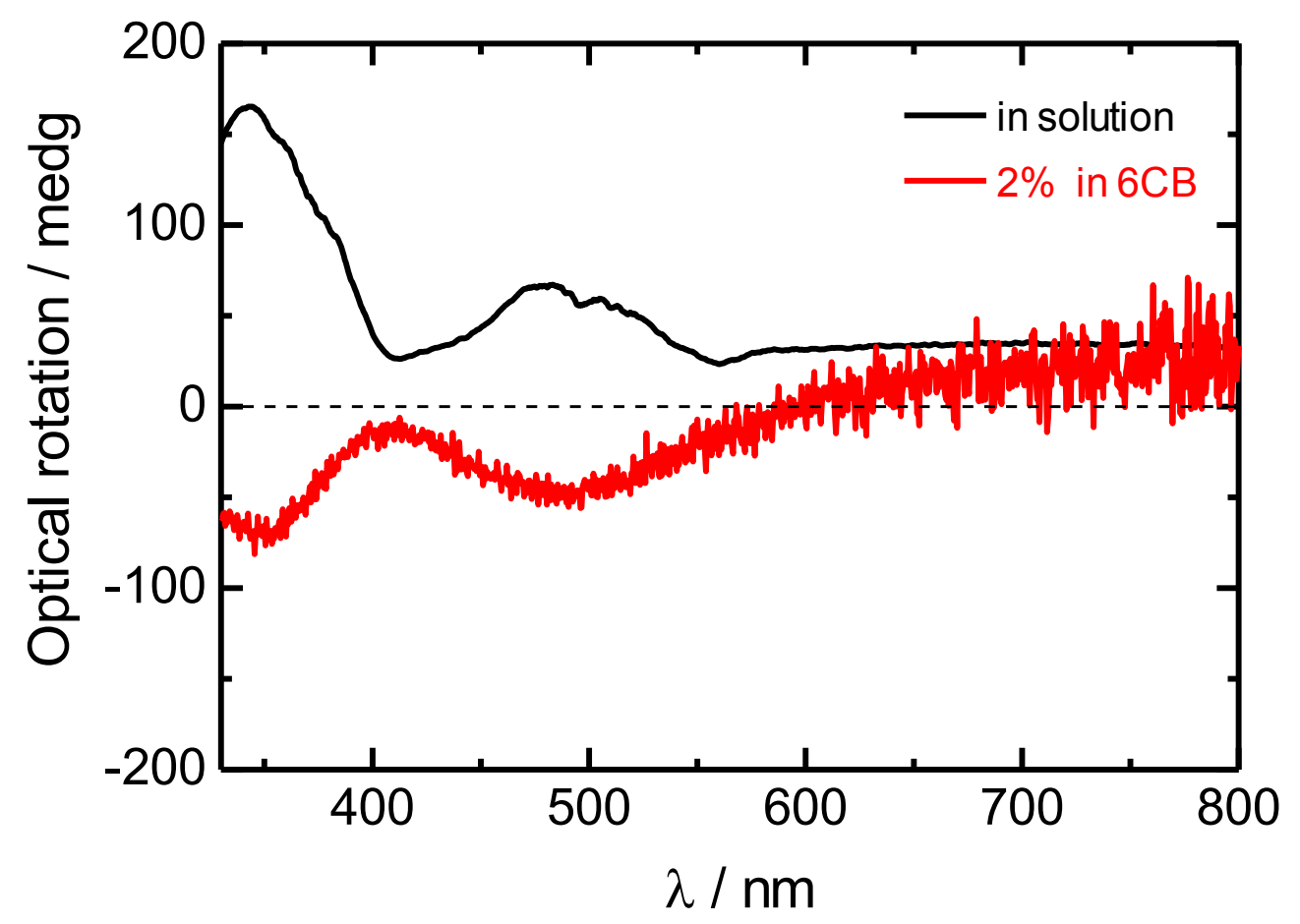

Fig. S5. ORD of compound $(S)$-D8Tt-B* in THF solution and 6CB. 
Synthesis and characterization of a novel donor-acceptor-donor chiral inducer and application in electrochemical polymerization

Jiuchao Dong, Kohsuke Kawabata, and Hiromasa Goto*

J. Mater. Chem. C, 2015,3, 2024-2032.

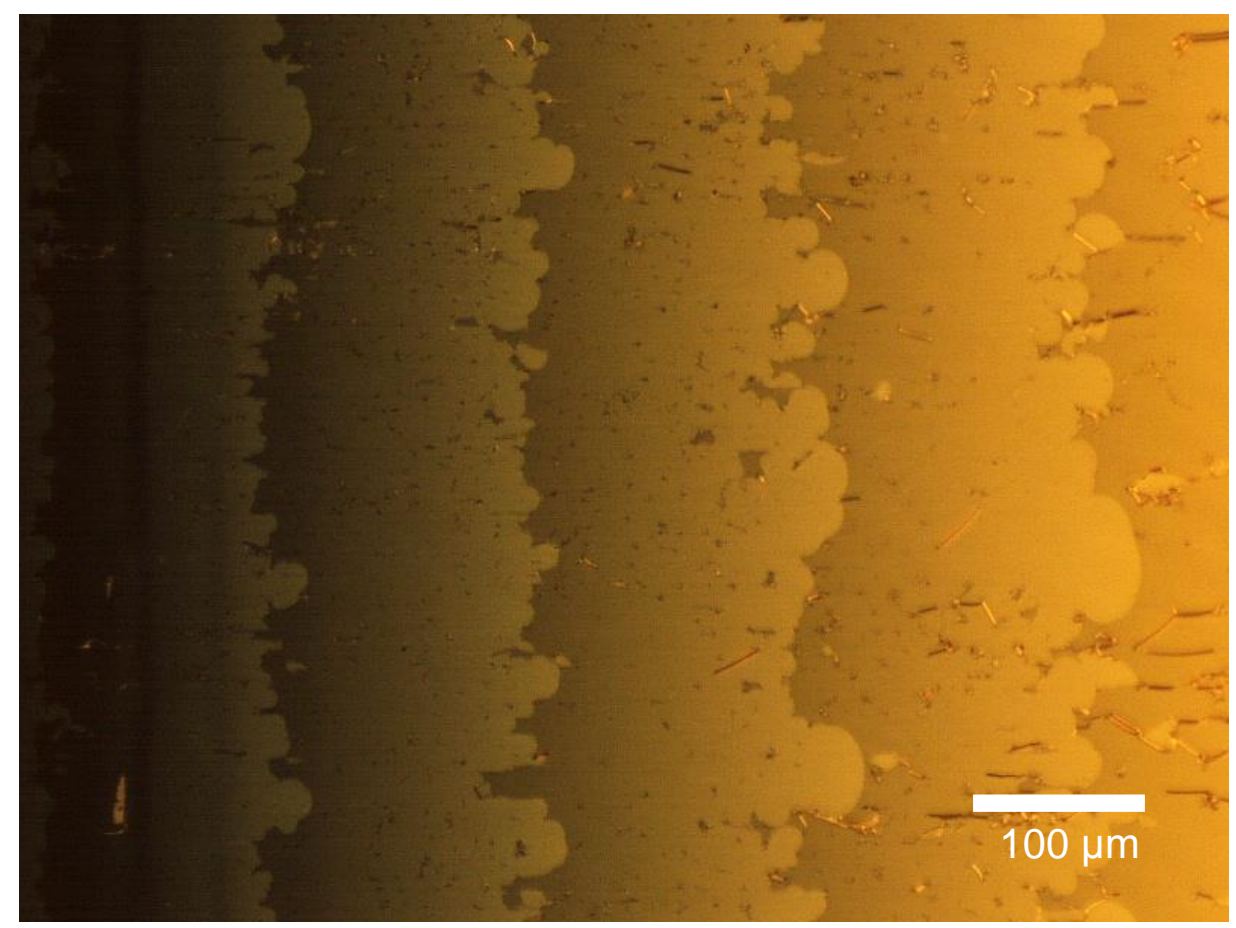

Fig. S6. POM image of the liquid crystal sample in the Grandjean-Cano cell. 
Synthesis and characterization of a novel donor-acceptor-donor chiral inducer and application in electrochemical polymerization

Jiuchao Dong, Kohsuke Kawabata, and Hiromasa Goto*

J. Mater. Chem. C, 2015,3, 2024-2032.

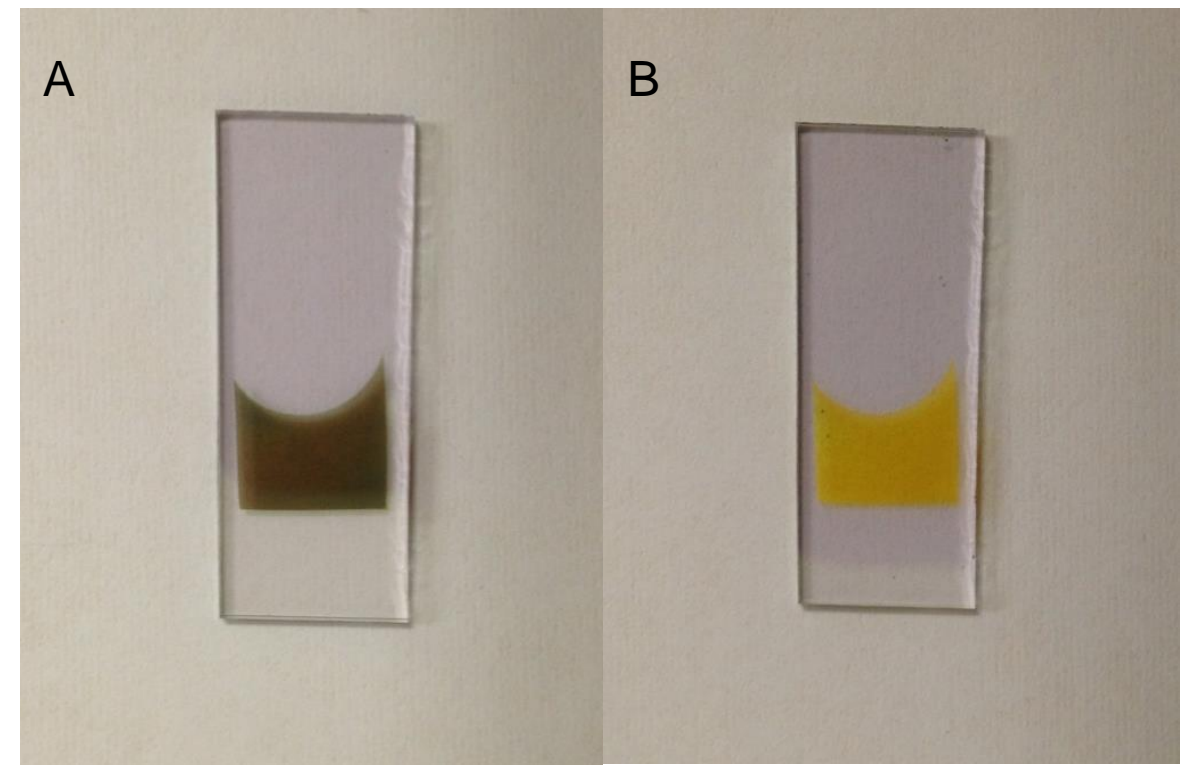

Fig. S7. Images of P-DFF films in oxidized state (A) and neutral state (B).

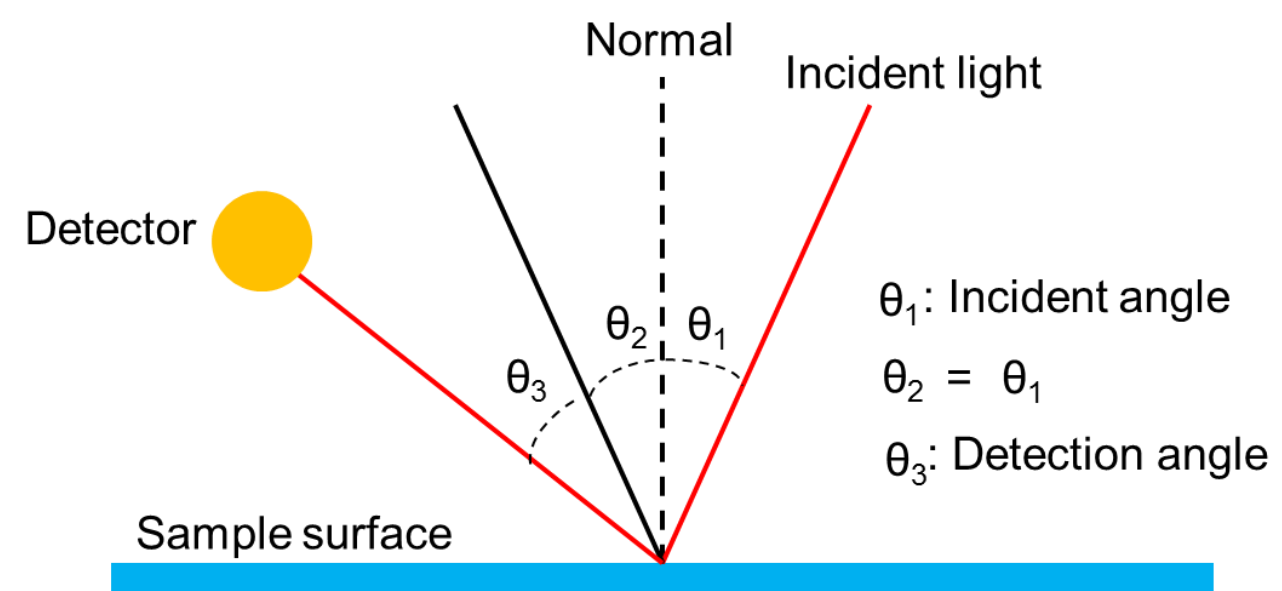

Fig. S8. Definition of incident angle and detection angle in reflection spectra. 
Synthesis and characterization of a novel donor-acceptor-donor chiral inducer and application in electrochemical polymerization

Jiuchao Dong, Kohsuke Kawabata, and Hiromasa Goto*

J. Mater. Chem. C, 2015,3, 2024-2032.

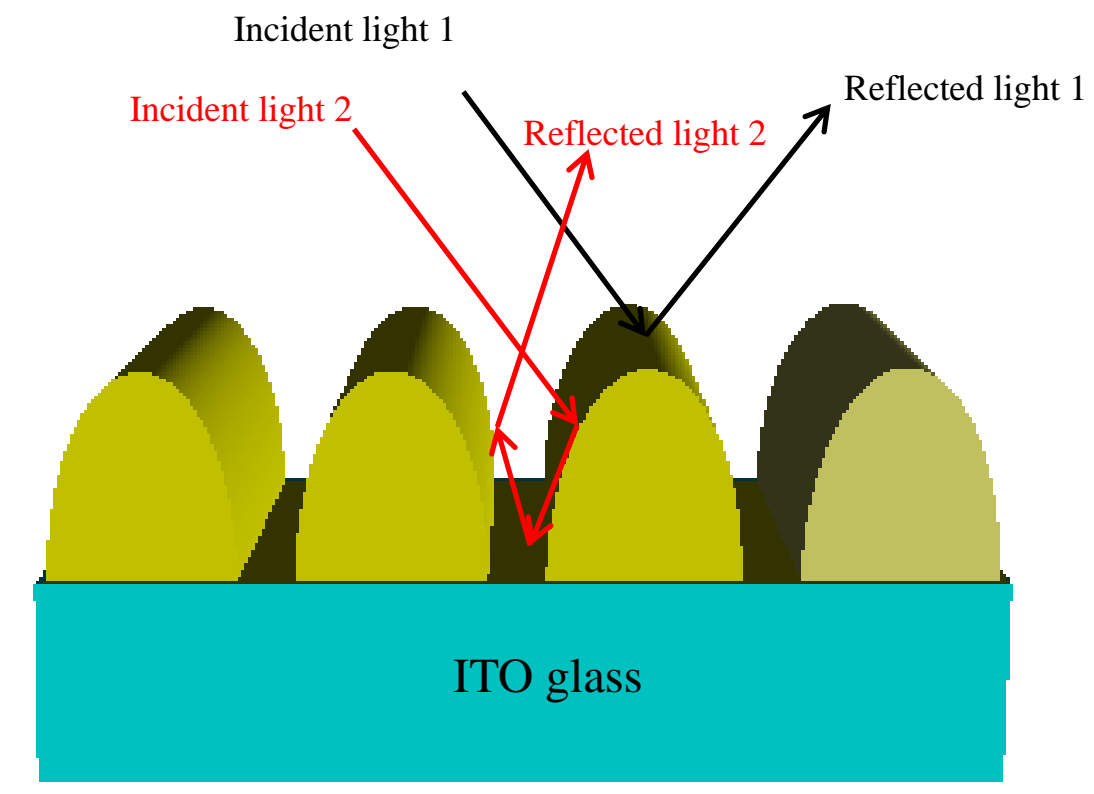

Fig. S9. Possible diagram of different reflection paths on the polymer film surface.

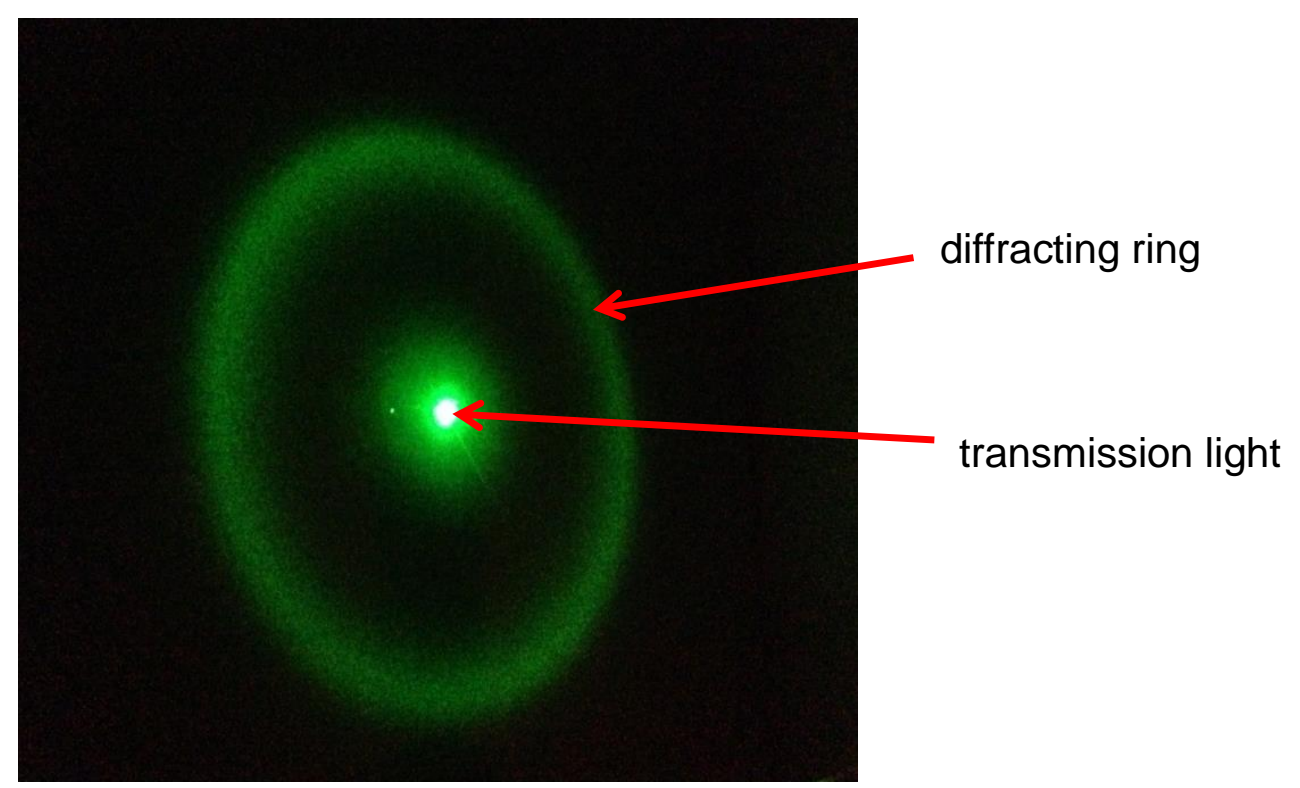

Fig. S10. Laser diffraction (532 nm). 